\title{
Low Dose GM-CSF Promotes Regeneration of CNS Axons
}

$$
\text { by }
$$

Jacqueline Legacy

A thesis submitted to the Faculty of Graduate and Postdoctoral Affairs in partial fulfillment of the requirements for the degree of

Master of Science

in

Neuroscience

Carleton University

Ottawa, Ontario

(C) 2012, Jacqueline Legacy 
Library and Archives

Canada

Published Heritage

Branch

395 Wellington Street

Ottawa ON K1A ON4

Canada
Bibliothèque et

Archives Canada

Direction du

Patrimoine de l'édition

395 , rue Wellington

Ottawa ON K1A ON4

Canada
Your file Votre référence

ISBN: 978-0-494-93586-6

Our file Notre référence

ISBN: 978-0-494-93586-6
NOTICE:

The author has granted a nonexclusive license allowing Library and Archives Canada to reproduce, publish, archive, preserve, conserve, communicate to the public by telecommunication or on the Internet, loan, distrbute and sell theses worldwide, for commercial or noncommercial purposes, in microform, paper, electronic and/or any other formats.

The author retains copyright ownership and moral rights in this thesis. Neither the thesis nor substantial extracts from it may be printed or otherwise reproduced without the author's permission.
AVIS:

L'auteur a accordé une licence non exclusive permettant à la Bibliothèque et Archives Canada de reproduire, publier, archiver, sauvegarder, conserver, transmettre au public par télécommunication ou par l'Internet, prêter, distribuer et vendre des thèses partout dans le monde, à des fins commerciales ou autres, sur support microforme, papier, électronique et/ou autres formats.

L'auteur conserve la propriété du droit d'auteur et des droits moraux qui protege cette thèse. $\mathrm{Ni}$ la thèse ni des extraits substantiels de celle-ci ne doivent être imprimés ou autrement reproduits sans son autorisation.
In compliance with the Canadian Privacy Act some supporting forms may have been removed from this thesis.

While these forms may be included in the document page count, their removal does not represent any loss of content from the thesis.
Conformément à la loi canadienne sur la protection de la vie privée, quelques formulaires secondaires ont été enlevés de cette thèse.

Bien que ces formulaires aient inclus dans la pagination, il n'y aura aucun contenu manquant. 


\section{Abstract}

Recent studies have shown the importance of granulocyte macrophage-colony stimulating factor (GM-CSF) as a potential therapeutic agent for central nervous system (CNS) injury. GM-CSF appears to mediate the injury response by promoting neuronal survival via the release of neurotrophic cytokines, and potentially promoting antiapoptotic mechanisms. Given the reactivity of the CNS following injury and recent evidence suggesting a developmental decline in CNS nerve regeneration, it is hypothesized that there is a possible beneficial role for GM-CSF in the treatment of CNS injury or illness. Experiments were therefore designed to assess the effect of GM-CSF administration on CNS axon growth and cell survival at different developmental stages. An in vitro retinal ganglion cell (RGC) culture system was used to determine whether GM-CSF plays a role in promoting axon growth, and the mechanisms underlying this effect were examined. The results reveal that GM-CSF promotes axon growth and cell survival in RGCs through activation of the mTOR (mammalian target of rapamycin) signaling pathway. 


\section{Acknowledgements}

First and foremost I would like to extend my appreciation and sincere gratitude to Dr. Patrice Smith for her encouragement and continual guidance throughout my time here at Carleton. Her knowledge and expertise has been invaluable. I would also like to thank my committee members, Dr. Alfonso Abizaid and Dr. John Stead for their insight into my thesis and for their continued support. A huge thanks goes out to Jennifer Theoret and Sonia Hanea for all of their help and patience - I couldn't have done it without you. I would like to thank Sonia in particular for the numerous hours she has spent helping me in the lab these last few months. A final thank you goes out to my friends and family who have always been there for me, and without whom, I couldn't have made it through. 


\section{Table of Contents}

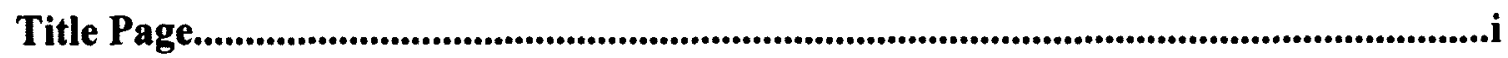

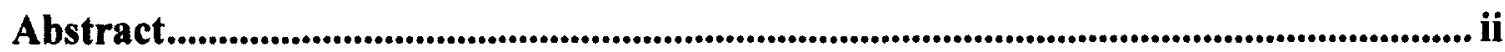

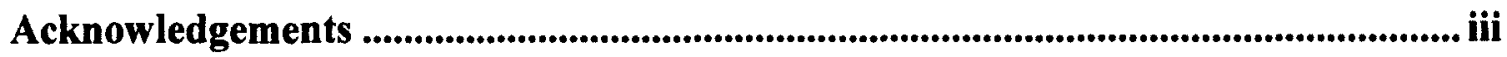

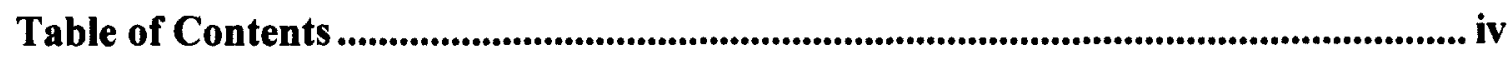

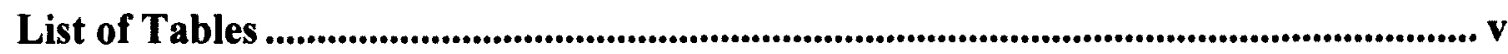

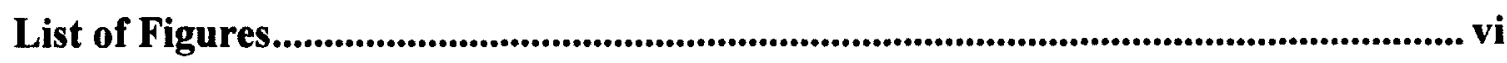

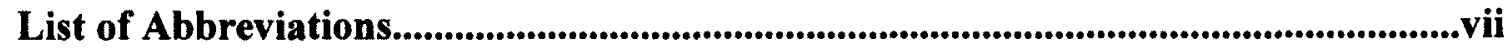

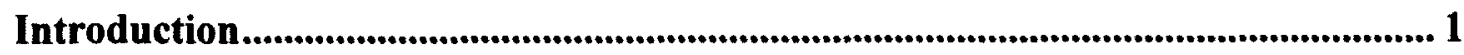

The Inhibitory Nature of the CNS Environment Following Injury..............................

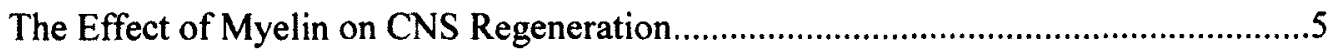

The Role of Microglia in CNS Regeneration......................................................

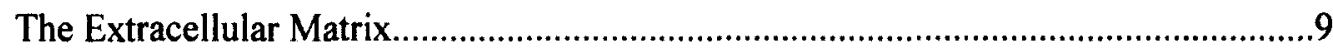

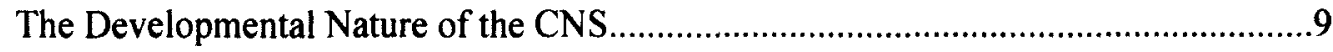

Granulocyte Macrophage-Colony Stimulating Factor (GM-CSF) .........................11

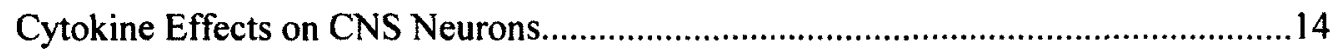

Determining GM-CSF's Role in CNS Regeneration.........................................18

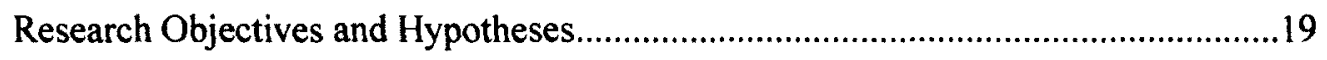

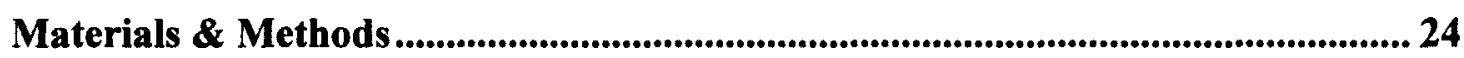

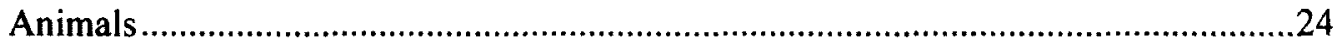

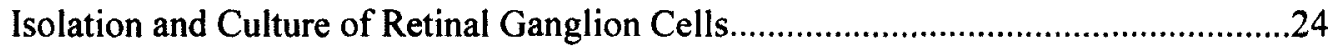

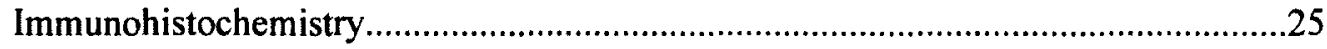

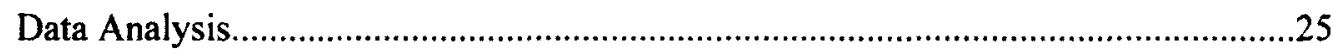

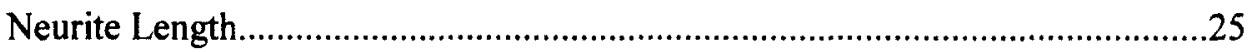

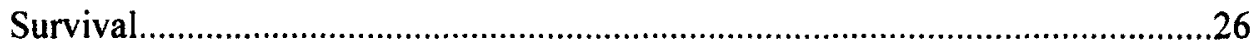




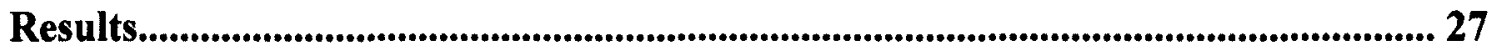

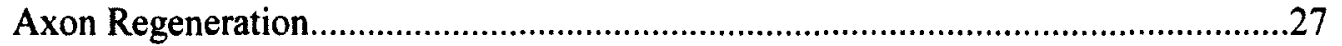

P6

P10

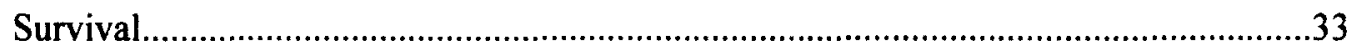

P6

Potential Mechanisms Mediating GM-CSF's Effects.....................................................37

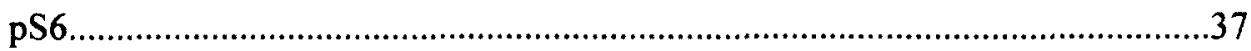

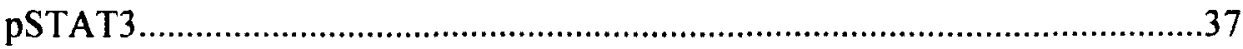

Discussion.................................................................................................................................................39

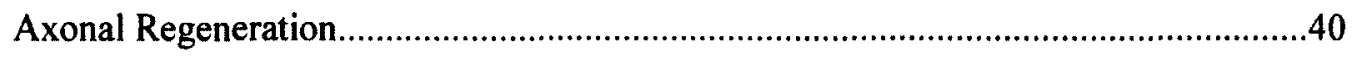

P6 -

P10

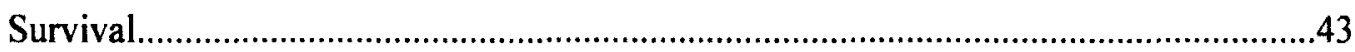

P6

P10

Signaling Mechanisms ...........................................................................................44

JAK/STAT Signaling ........................................................................................

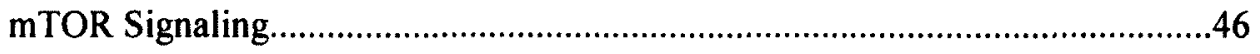

SOCS3 \& PTEN.......................................................................................

Conclusions \& Future Directions..........................................................................49

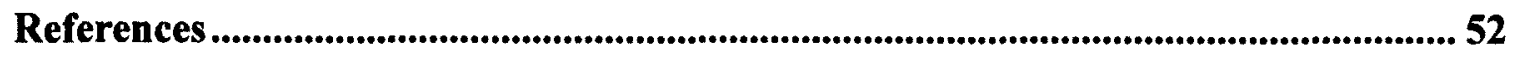




\section{List of Tables}

Table 1. Mean Differences in Neurite Length $(\mu \mathrm{m})$ Expressed as a Function of

Postnatal Date and Treatment Group

Table 2. Mean Differences in Cell Count Expressed as a Function of Treatment Group.34 


\section{List of Figures}

Figure 1. JAK/STAT, PI3K/AKT, MAPK \& mTOR Signaling Cascade................... 16

Figure 2. Culture Plate Treatment Group Design ................................................. 23

Figure 3. Survival Images Taken Across Each Well...............................................26

Figure 4. Mean Neurite Length of Postnatal Day 6 RGCs in Culture...........................30

Figure 5. Mean Neurite Length of Postnatal Day 10 RGCs in Culture.........................30

Figure 6. Regeneration of Postnatal Day 6 RGCs in Culture......................................

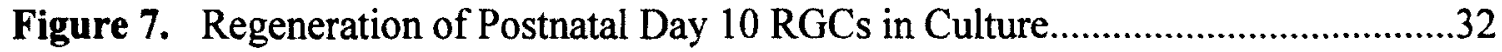

Figure 8. Mean Cell Count as an Indicator of Survival in Postnatal Day 6 RGCs.........35

Figure 9. Survival Effect in GM-CSF Treated Postnatal Day 6 RGCs........................36

Figure 10. Immunostaining of pS6 in Postnatal Day 6 RGCs in Culture........................38

Figure 11. mTOR Signaling Mechanism..........................................................47 


\section{List of Abbreviations}

ANOVA - analysis of variance

BDNF - brain derived neurotrophic factor

cAMP - cyclic adenosine monophosphate

CNS - central nervous system

CNTF - cilliary neurotrophic factor

CSPG - chondroitin sulphate proteoglycan

DMEM - Dulbecco's modified Eagle's medium

DPBS - distilled phosphate buffered saline solution

FBS - fetal bovine serum

GM-CSF - granulocyte-macrophage colony-stimulating factor

HBSS - Hank's buffered saline solution

IL-6 - interleukin 6

JAK/STAT - Janus kinase-signal transducer and activator of transcription

JAK2 - Janus kinase 2

LPC - lysophosphatidylcholine

MAG - myelin associated glycoprotein

MAPK - mitogen-activated protein kinase

mTOR - mammalian target of rapamycin

NGF - neurotrophic growth factor

NT3 - neurotrophic factor 3

OMgp - oligodendrocyte myelin glycoprotein

PBS - phosphate buffered saline solution 
PI3K - phosphoinositide 3-kinase

PNS - peripheral nervous system

pSTAT3 - phospho-signal transducer and activator of transcription 3

PTEN - phosphatase and tensin homologue

RGMa - repulsive guidance molecule A

RGC - retinal ganglion cell

SOCS3 - suppressor of cytokine signaling 3 


\section{INTRODUCTION}

During development, the central nervous system (CNS) exhibits an extremely growth permissive environment (Benowitz \& Yin, 2010; Coleman, 2005; Lim \& Tow, 2007; Muramatsu, Ueno, \& Yamashita, 2009). Growth factors such as brain derived neurotrophic factor (BDNF), neurotrophic factor 3 (NT3) and nerve growth factor (NGF) encourage neuritic and vascular growth, and axon guidance molecules direct growing axon fibers to connect with appropriate targets (Harel \& Strittmatter, 2006). Sensory experience drives the formation of new synapses and neural circuits are remodeled as the brain strengthens active connections and eliminates others (Thuret, Moon, \& Gage, 2006; Yiu \& He, 2006).

As CNS development nears completion however, growth promoting signals and pathways are down regulated and many in fact change their function to inhibit growth within the mature CNS (Muramatsu et al., 2009; Tanaka \& Ferretti, 2009; Yiu \& He, 2006). While this transition signals the end of development, and is necessary to prevent overgrowth and improper synaptic connectivity, this loss of intrinsic regenerative ability that coincides with the end of development has been found to limit regeneration of damaged axons in the adult brain and spinal cord. Whenever the CNS becomes damaged through illness or traumatic injury, cell bodies as well as the processes of neurons can become damaged without the ability to be replaced (Thuret et al., 2006). In fact, one of the major characteristics of neurological trauma associated with spinal cord injury, stroke and several neurodegenerative disorders is a dramatic loss of neurons and axonal 
projections within the CNS (Thuret et al., 2006). This can result in devastating consequences on neurological function.

Thousands of Canadians suffer from traumatic brain and spinal cord injuries every year (Thuret et al., 2006). With no viable treatments available, these individuals, and many others who suffer from stroke or neurodegenerative disorders, often experience a dramatic decline in both cognitive and physical functioning, and ultimately face a lifetime of illness and supportive care (Ha et al., 2005; Thuret et al., 2006). These devastating injuries and illnesses not only impact the lives of Canadians and their families; they have tremendous implications for the Canadian health care system and economy. Cost of care for individuals with neurological trauma can be enormous, especially when young Canadians are affected. Therefore, finding viable treatment options that can promote CNS regeneration and functional recovery, will help alleviate the pressure that is placed on the health care system, and give affected Canadians a second chance at life.

Concerted research effort has been geared toward understanding the mechanisms that mediate the lack of repair that occurs within the damaged CNS. Following injury there is an extensive loss of neurons in and around the site of injury and often throughout the brain (Rotshenker, 2011; Shibuya, Yamamoto, \& Itano, 2009). Furthermore, it is believed that the lack of axonal regeneration that occurs in the brain and spinal cord is due in large part to the reactive changes that occur at the lesion site, and to the developmental loss of regenerative ability that takes place in the mature CNS (Rotshenker, 2011). This lack of intrinsic regenerative ability has been researched extensively, with previous evidence indicating that the inhibitory CNS environment plays 
a major role in the loss of axon growth ability that is seen following injury in the mature CNS. Interestingly, GM-CSF (granulocyte macrophage-colony stimulating factor), a hematopoietic cytokine typically used in the treatment of oncologic disorders, has been shown to counteract this developmental increase in CNS inhibition and promote healing and regeneration in in vivo models of optic nerve crush and spinal cord injury (Bouhy et al., 2006; Fathali, Lekic, Zhang, \& Tang, 2010; Kim et al., 2009; Kim et al., 2012; Mangano et al., 2011). This suggests a possible role for GM-CSF in the treatment of CNS injury. However, while select studies have now shown that GM-CSF has the ability to promote neuronal survival following injury, the mechanisms underlying these regenerative effects have yet to be elucidated. The objective of this thesis is to evaluate the regenerative capabilities of GM-CSF in an established, in vitro model of CNS injury, and more specifically, to determine the molecular mechanisms underlying the hypothesized GM-CSF mediated effects.

\section{The Inhibitory Nature of the CNS Environment Following Injury}

Following injury, the CNS undergoes a series of reactive changes. Injury or illness can damage both the cell bodies and processes of neurons and may even lead to extensive cell loss throughout the brain and spinal cord (Sun \& He, 2010). When neuronal cells in the brain become damaged, distal axon segments retract from their postsynaptic connections and undergo a process called Wallerian degeneration (Gaudet, Popovich, \& Ramer, 2011). While the proximal segments of axons often survive, they are rarely seen to spontaneously regenerate. The myelin structure that ensheaths nerve fibers may also begin to deteriorate, leaving severed axon segments vulnerable to myelin- 
associated inhibitors and other molecules that are detrimental to axonal regeneration (Gaudet et al., 2011).

In response to this mechanical and chemical damage, supportive cells such as macrophages, astrocytes and microglia, gather at the lesion site to trigger an inflammatory response within the CNS (Gaudet et al., 2011; Huebner \& Strittmatter, 2009; Muramatsu et al., 2009). While research has shown that inflammation is a way for the CNS to try to heal itself, the inflammatory process itself can often have negative effects in and around the site of injury (Huebner \& Strittmatter, 2009).

As reactive astrocytes gather around the lesion site, they form a glial scar that acts as a physical and chemical barrier to regeneration (Buffo, Rolando, \& Ceruti, 2010; Thuret et al., 2006). This layer of tissue often develops perpendicular to the lesion site and is impenetrable to sprouting axons, due not only to its fibrous structure, but also to the inhibitory molecules that it secretes into the extracellular matrix (Silver \& Miller, 2004; Sofroniew, 2009; Yiu \& He, 2006)

As inflammatory cells invade the lesion site, the extracellular matrix is infiltrated by several different types of inhibitory molecules (Buffo et al., 2010; Yiu \& He, 2006). Chondroiton sulphate proteoglycan (CSPG) expression is rapidly up regulated by reactive astrocytes after injury, forming an inhibitory gradient that is highest at the center of the lesion site; and inflammatory cytokines are recruited to the site of injury, inhibiting axon growth and promoting growth cone collapse (Fitch \& Silver, 2008; Peterson, Wang, Tzekova, \& Wiegand, 2000).

Despite the abundance of evidence indicating that CNS axons are simply unable to regenerate following injury, a classic study carried out by David \& Aguayo (1981) 
showed that CNS axons can regenerate within peripheral nerve grafts. This indicates that although the CNS experiences a developmental decline in axon growth ability, CNS axons are indeed able to regenerate; however, they require an environment that is both well structured and permissive to growth in order to do so (Richardson, McGuinness, \& Aguayo, 1980). Therefore, up-regulating signals that are active during development and which increase the regenerative capacity of mature CNS axons may be an important factor when designing effective treatments for CNS injury. Because the inhibitory CNS environment has also been shown to play a major role in the lack of regeneration that is seen following injury, combinatorial therapies that both increase intrinsic the regenerative capability of axons and decrease extrinsic inhibitory factors within the CNS environment, will likely be most effective when treating brain and spinal cord injuries. Further analysis of the injury response will shed light on both the intrinsic and extrinsic factors that influence CNS regeneration.

\section{The Effect of Myelin on CNS Regeneration}

Myelin associated inhibitors have an important role in the lack of regeneration that is seen in the adult CNS following injury. As damaged axons undergo Wallerian degeneration, there is often a breakdown of axonal fibers (Ousman \& David, 2001; Rotshenker, 2011). The myelin sheath surrounding the fibers can deteriorate and while both microglia and macrophages are recruited to clear the extracellular matrix of myelin and axonal debris, this process is often quite slow, allowing myelin associated inhibitors, such as myelin associated glycoprotein (MAG), oligodendrocyte-myelin glycoprotein (OMgp) and NogoA, to surround fibers and further hinder regenerative processes (Gaudet 
et al., 2011; Ousman \& David, 2001; Rotshenker, 2011). Another barrier to regeneration is the inability of oligodendrocytes to remyelinate damaged nerve fibers following injury. Oligodendrocytes often undergo apoptosis or enter a quiescent state following injury, rendering them unable to remyelinate damaged axonal fibers (Gaudet et al., 2011; Ousman \& David, 2001). They are also unable to engulf debris efficiently, and provide little growth support to surrounding neurons (Gaudet et al., 2011).

While all of these factors have a role in creating an inhibitory CNS environment following injury, it is important to note that eliminating myelin inhibition is not sufficient to promote axonal regeneration in the mature CNS. Lee and colleagues (2010) carried out several studies that assessed axon regeneration in Nogo, MAG and OMgp deficient mice, and found that while individual deletion of each of the aforementioned inhibitors induced sprouting of corticospinal axons, triple knockout mice failed to exhibit robust axon regeneration following spinal cord injury (Lee, Chow, et al., 2010; Lee, Geoffroy, et al., 2010). Several other studies have also shown that although Nogo, MAG and OMgp appear to contribute to the inhibitory CNS environment following injury, deletion of all three myelin inhibitors and their receptors does not enhance axonal regeneration following injury (Fischer, 2010). This suggests the possibility of other contributing factors, such as those responsible for the developmental loss of axon growth ability.

\section{The Role of Microglia in CNS Regeneration}

Activated microglia and macrophages produce a major inhibitory environment that limits CNS regeneration (Kitayama, Ueno, Itakura \& Yamashita, 2011). Both microglia and macrophages express the inhibitory guidance molecules Slit, Netrin-1, and repulsive guidance molecule $\mathrm{A}$ ( $\mathrm{RGMa}$ ), which have been shown to repel extending 
axons and promote the collapse of growth cones following injury (Kitayama et al., 2011). RGMa expression is particularly up-regulated after injury, with inhibition of this molecule associated with enhanced axonal growth and motor function recovery in in vivo studies of axonal growth inhibition (Kitayama et al., 2011). By inducing the retraction of dystrophic axons, microglia and macrophages increase the dieback of injured axons at the site of injury, reducing the chances of successful regeneration (Kitayama et al., 2011).

While macrophages and microglia often appear to hinder CNS regeneration, in many circumstances, they are also responsible for a degree of the recovery that is seen at the site of injury. One reason for this dichotomous view of microglia and macrophages lies in the generalization of subsets of macrophages and microglia, into one category of inflammatory cells (Kitayama et al., 2011). In actuality, both microglia and macrophages express two differing phenotypes. There are classically activated macrophages and microglia (M1), which are responsible for secreting high quantities of pro-inflammatory cytokines and free radicals; and alternatively activated macrophages and microglia (M2), which are responsible for reducing inflammation and repairing damaged tissue (Kitayama et al., 2011).

While macrophages contribute to the extracellular environment, it is the classically activated microglia that are primarily responsible for the axonal growth inhibition that is seen following injury (Kitayama et al., 2011). While M2 microglia are essentially neuroprotective, promoting healing of the lesion site and allowing axonal growth to occur, their M1 counterparts appear to be neurotoxic, reducing the regenerative capacity of damaged axons (Kitayama et al., 2011). Since both subtypes of microglia are up-regulated following traumatic injury, it is likely the ratio of M1/M2 expression that 
determines whether microglia have a neuroprotective or neurotoxic effect on axons (Kitayama et al., 2011).

Unfortunately, M1 expression tends to become up-regulated over the course of CNS development, ultimately reducing the regenerative capacity of neurons. This is in contrast to the peripheral nervous system (PNS), where the M2 subtype of microglia predominates as times goes on, facilitating nerve regeneration and functional recovery (Kitayama et al., 2011). Enhanced M2 expression within the PNS not only promotes angiogenesis and continued healing at later stages of recovery, it also facilitates the release of anti-inflammatory cytokines, which in turn leads to extensive and efficient axon growth (Gaudet et al., 2011).

Another factor influencing the ability of the CNS to regenerate following injury is the efficiency with which macrophages within the CNS are able to remove axonal and myelin debris from the injury site. While Schwann cells are able to quickly remove myelin debris from the PNS following Wallerian degeneration, neither macrophages nor oligodendrocytes are able to efficiently remove debris from the lesion site, in turn leaving sensitive axon fibers exposed to myelin-associated inhibitors (Gaudet et al., 2011; Ousman \& David, 2001).

Axonal and myelin debris can also physically interfere with axons trying to extend past the site of injury. Advancing axon fibers are guided by growth cones composed of microtubules, which extend into the periphery to detect extracellular guidance cues (Hur et al., 2011). Following injury, these microtubules can become damaged and disorganized as injured axons form retraction bulbs and undergo Wallerian degeneration (Hur et al., 2011). Axonal and myelin debris can further impede function of 
these already damaged microtubules, preventing them from properly detecting molecular signals within the matrix (Hur et al., 2011).

\section{The Extracellular Matrix}

While the secondary injury response heavily contributes to the lack of regeneration that is seen in the CNS, the structure of the extracellular matrix itself acts as a barrier to regeneration. The extracellular matrix often lacks sufficient levels of collagen, laminin and fibronectin, all of which contribute to the strength and structure of the CNS (Gaudet et al., 2011). As a result, regenerating CNS axons are at a disadvantage, and may have difficulty extending over the physical and chemical terrain of the lesion site. Oligodendrocytes within the CNS also do not have an associated basal lamina, a structure that is an integral part of the PNS extracellular environment and that is extremely important to PNS nerve regeneration (Gaudet et al., 2011).

\section{The Developmental Nature of the CNS}

Recent hypotheses suggest that unlike PNS neurons, CNS neurons undergo a permanent down-regulation of axon growth potential during development (Newbern, Shoemaker, \& Snider, 2009). Signaling pathways that promote axonal growth and connectivity are extremely active during development but eventually decrease their activity as sufficient connections are formed and development nears completion (Newbern, Shoemaker \& Snider, 2009; Park et al., 2008). Other pathways may also up regulate their activity to prevent overgrowth and to allow for synaptic remodeling of neural circuits in the brain (Park et al., 2008). As a result, it has been hypothesized that inhibiting particular signaling pathways in the brain that promote cell growth and 
proliferation during development but inhibit growth as development nears completion may lead to regeneration in the mature CNS (Newbern et al., 2009; Smith et al., 2009). Up-regulating the expression of growth factors that are vital during development may also promote a more growth permissive environment in the adult (Kurimoto et al., 2010).

Given that growth and regeneration is possible in the human embryonic nervous system, a goal of the current research is to fully understand how the adult CNS differs from that of the developing CNS, so that therapies can be designed to enhance mechanisms in the adult that have been shown to be important during development. For example, mature CNS neurons, like embryonic and PNS neurons, express regeneration associated genes following injury (Harel \& Strittmatter, 2006). Adult CNS neurons however, do not have the ability to fully translate these signals into a successful regenerative response because they lack the downstream effectors that are required for signal translation (Harel \& Strittmatter, 2006). Mature neurons also express reduced levels of cAMP, and increased levels of the signal transduction machinery that processes inhibitory extracellular molecules (Harel \& Strittmatter, 2006). This leads to altered neuronal activity and greater sensitivity to extracellular inhibition, compared to embryonic or PNS neurons.

Because adult CNS neurons are more sensitive to the inhibitory and neuroinflammatory processes that occur following injury, developing treatments that reduce inhibitory factors and neuroinflammation is critical. Enhancing regenerative signals that are active during development may also enhance cell survival and axonal regeneration at later developmental time points. Discovering and understanding therapeutic agents that are multifaceted in their regenerative capabilities, such as GM- 
CSF, will be of particular importance, as it appears that multiple mechanisms may be involved in priming the mature CNS to produce a successful regenerative response following injury.

\section{Granulocyte-Macrophage Colony Stimulating Factor (GM-CSF)}

Granulocyte macrophage-colony stimulating factor (GM-CSF) is a hematopoietic cytokine that is often used to stimulate bone marrow stem cell proliferation in individuals with hemato-oncologic disorders (Kim et al., 2009; Zhan, Xu \& Lew, 2012). It has been shown to prevent apoptosis of hematologic cells and to trigger an immune response following injury, stimulating the proliferation and differentiation of myeloid progenitors, neutrophil precursors, monocytes and macrophages, and recruiting these cells to form a host response (Hayashi, Ohta, Kawakami, \& Toda, 2009; Kim et al., 2009; Manczak et al., 2009; Qin, Holdbrooks, Liu, Reynolds, Yanagisawa \& Benveniste, 2012; Reddy et al., 2009).

While GM-CSF has traditionally been recognized for its role in the treatment of oncologic disorders, there is a possible role for this inflammatory cytokine as a therapeutic agent for CNS damage and degeneration. Importantly, GM-CSF has receptors in both the brain and spinal cord, (as well as in RGCs, neural progenitor cells, oligodendrocytes and microglia) and is able to cross the blood-brain barrier, whereas other trophic factors are not (Bouhy et al., 2006; Mangano et al., 2011; McLay, Kimura, Banks \& Kastin, 1997; Ousman \& David, 2001; Reddy et al., 2009; Ridwan, Bauer, Frauenknecht, von Pein \& Sommer, 2012; Schallenberg, Charalambous \& Thanos, 2009). GM-CSF has also been shown to enhance the injury response by increasing microglial activation, inhibiting gliosis and promoting the release of neurotrophic cytokines such as 
BDNF (Bouhy et al., 2006; Mangano et al., 2011; Ousman \& David, 2001). This leads to enhanced neural protection, with GM-CSF actually promoting functional recovery in patients following spinal cord injury and stroke (Park et al., 2005; Yoon et al., 2007). Additionally, GM-CSF has been shown to increase the activation of endogenous neural stem cells and to guide these cells as they differentiate and migrate to damaged neural regions (Stosser, Schweizerhof, \& Kuner, 2011). This may be of particular importance in cases where there is progressive neurological deterioration.

Several studies have looked at the effects of GM-CSF on CNS regeneration. One such study carried out by Kim and colleagues (2009), looked at the effects of GM-CSF on glial scar formation in an in vivo model of spinal cord injury. The results showed that GM-CSF administration significantly reduces cavity size and glial scar formation 3-4 weeks following spinal cord injury (Huang et al., 2009). They also found that GM-CSF treatment decreases CSPG expression and helps to preserve the axonal arrangement and myelin structure within the lesion site (Huang et al., 2009).

Another study looking at GM-CSF's involvement in the immune response that follows CNS injury, showed that GM-CSF mediates the rapid recruitment of monocytes, neutrophils, $T$ cells and macrophages, that occurs following injury (Ousman \& David, 2001). GM-CSF also attenuates the effects of the demyelinating agent, lysophosphatidylcholine (LPC), on the adult mouse spinal cord by recruiting microglia and macrophages, and induces rapid phagocytosis of broken down myelin from the CNS (Ousman \& David, 2001). Although GM-CSF is naturally released by astrocytes, T cells and other immune related cells, GM-CSF treatment has the ability to further enhance the immune response by stimulating myelin clearance during Wallerian degeneration 
following brain and spinal cord injury, and in neurodegenerative disorders such as multiple sclerosis (Ousman \& David, 2001).

Finally, there have been several studies outlining GM-CSF's usefulness as a promoter of neuronal survival in several animal models of optic nerve crush, spinal cord injury, stroke and Parkinson's disease (Bouhy et al., 2006; Fathali, Lekic, Zhang, \& Tang, 2010; Kim et al., 2009; Mangano et al., 2011). Given the nature of these studies and the fact that GM-CSF appears to modulate the injury response to promote neuronal survival, there is a possible role for GM-CSF in the treatment of brain and spinal cord injury, stroke and other neurodegenerative disorders.

Interestingly, several studies have indicated that GM-CSF may mediate the injury response by modulating three main signaling pathways: the Janus kinase-signal transducer and activator of transcription (JAK/STAT) pathway, the mitogen-activated protein kinase (MAPK) pathway, and the phosphoinositide 3-kinase (PIK3) pathway (Figure 1) (Goodwin, Yang, Yin, Yu \& Chan, 2012; Schallenberg, Charalambous \& Thanos, 2009; Stosser et al., 2011). JAK/STAT signaling is quite important to CNS regeneration and leads to activation of the STAT family of transcription factors. STAT3, a regulator of gene transcription, in particular has been shown to be rapidly phosphorylated following GM-CSF activation (Bareyre et al., 2011; Stosser et al., 2011). Interestingly, STAT3 appears to have a major role in CNS regeneration, with elevated levels of STAT3 expression and phosphorylation associated with both axonal regeneration and synaptic remodeling in the brain and spinal cord (Bareyre et al., 2011; Stosser et al., 2011). Although STAT3 appears to be at least partially responsible for initiating axon regeneration following injury, it does not continue to perpetuate growth at 
later time points (Bareyre et al., 2011). Furthermore, although STAT3 deletion is enough to impair nerve regeneration within the PNS, once regeneration has been initiated, STAT3 deficient axons grow at the same speed as STAT3 competent axons (Bareyre et al., 2011). This supports the idea that STAT3 acts to jump-start the regeneration process following injury.

While GM-CSF activation of the JAK/STAT signaling pathway may initiate axon regeneration following injury by inducing phosphorylation of STAT3 (Bareyre et al., 2011), it is clear that several other signaling pathways and molecules must be involved in the GM-CSF mediated effects. GM-CSF has been shown to enhance cell survival, as well as initiate axonal regeneration in the damaged adult CNS, leading to the hypothesis that several different signaling pathways are likely activated by GM-CSF and may work synergistically to enhance regeneration (Bouhy et al., 2006; Huang et al., 2009; Mangano et al., 2011; Vassiliou et al., 2010).

\section{Cytokine Effects on CNS Neurons}

Interleukin-6 (IL-6) is a cytokine with both inflammatory and anti-inflammatory functions. Following axotomy, IL-6 expression is up-regulated within the CNS, inducing a gp130 dependent signaling cascade that ultimately results in the activation of the JAK/STAT3 signaling pathway (Sun \& He, 2010). Ciliary neurotrophic factor (CNTF) is another molecule that acts via a gp 130 dependent JAK/STAT signaling mechanism. Like Il-6, CNTF is up-regulated in the mature CNS following injury and acts to ultimately induce the phosphorylation of STAT3, indicating that CNTF likely has a role in initiating axon regeneration following injury (Smith et al, 2009). Several studies have now shown that CNTF expression is increased following optic nerve crush in mice (Fischer, 2010; 
Leibinger et al., 2009; Newbern et al., 2009; Peterson et al., 2000; Smith et al., 2009). One particularly interesting study however, carried out by Leibinger and colleagues (2009) showed that the enhanced CNTF expression that is seen following optic nerve crush and lens injury is paralleled by increased levels of pSTAT3 in retinal astrocytes in mice. This not only supports the idea that there is a direct and linear relationship between CNTF expression and levels of pSTAT3 following injury; it also provides evidence that CNTF release following injury may be glial-dependent. This suggests that although reactive astrocytes contribute to the inhibitory $\mathrm{CNS}$ environment following injury, they may also have a beneficial role in promoting axon regeneration at the lesion site.

While IL-6 and CNTF have both been shown to regulate regeneration via STAT3dependent mechanisms, suppressor of cytokine signaling 3 (SOCS3) also appears to be heavily involved in regulating the initiation of axon regeneration within the damaged CNS (Smith et al., 2009; Sun et al., 2011). A recent study carried out by Sun and colleagues (2011) found that sustained axon regeneration could be induced by co-deletion of both SOCS3 and PTEN. PTEN (phosphatase and tensin homologue), is a negative regulator of both the PI3K and the mammalian target of rapamycin (mTOR) signaling pathways (Kang, Lemke, \& Kim, 2009; Liu et al., 2010). PTEN has been shown to reduce the regenerative capacity of CNS neurons, with PTEN deletion increasing regenerative capacity via enhanced phosphorylation of $\mathrm{S} 6$, a key regulator of protein translation (Kurimoto et al., 2010; Sun et al., 2011). pS6 is critical to CNS regeneration in vivo (Kurimoto et al., 2010), and because it is regulated by the PTEN/mTOR, PI3K and MAPK signaling pathways, it would appear that GM-CSF could ultimately be 
triggering regeneration via downstream phosphorylation mechanisms involving both

STAT3 and S6 (Figure 1) (Kurimoto et al., 2010; Sun et al., 2011).

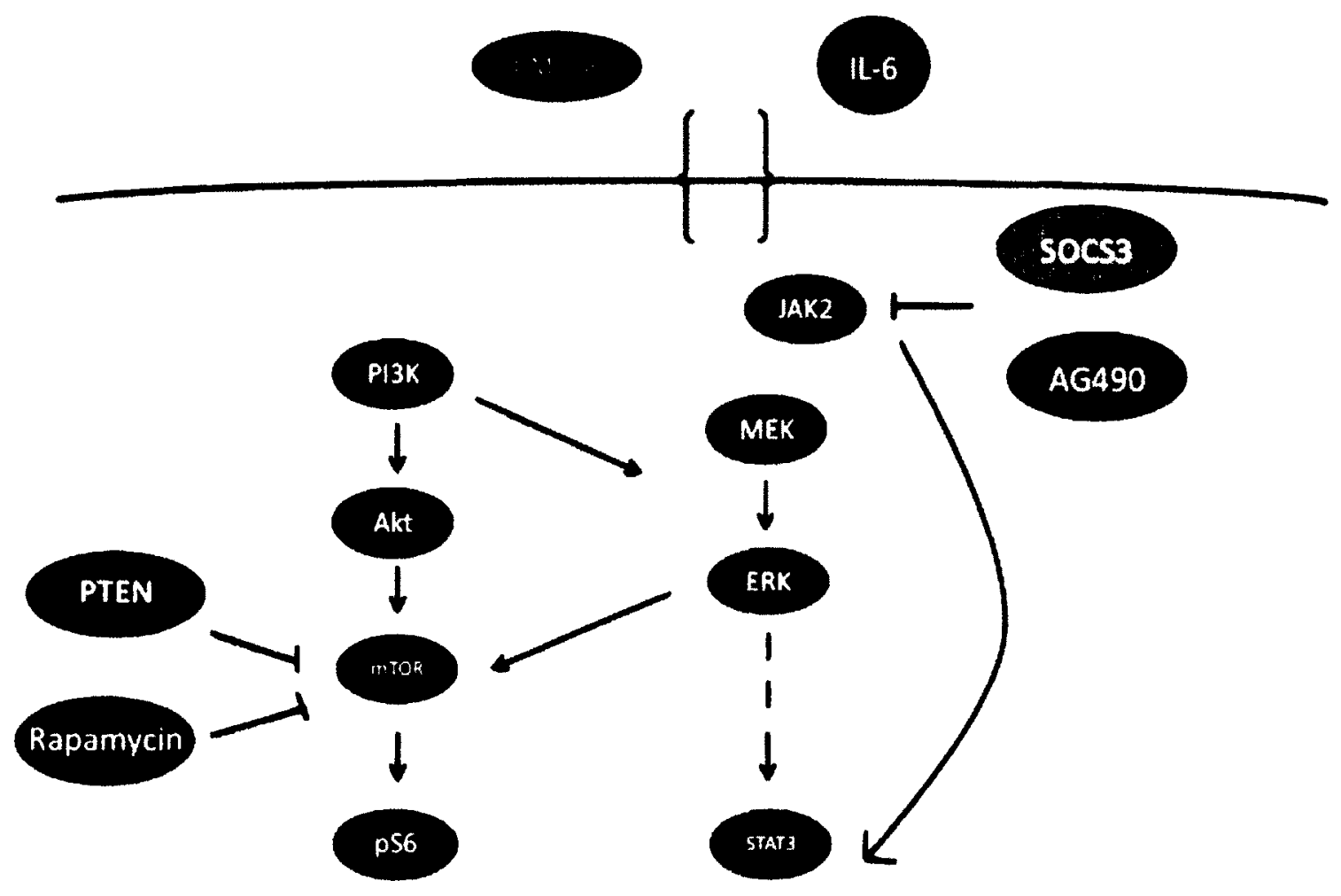

Figure 1. JAK/STAT, PI3K/AKT, MAPK \& mTOR Signaling Cascade. Extracellular activation of GM-CSF has the potential to trigger a response from several molecules within this signaling cascade. It is hypothesized that GM-CSF may promote regeneration either via mTOR or JAK2/STAT3 signaling, culminating in the phosphorylation of S6 and STAT3 respectively. While STAT3 phosphorylation is thought to jump-start regenerative processes following injury, phosphorylation of S6 is associated with increased levels of protein translation and a prolonged regenerative response within the CNS. 
Although GM-CSF may activate any one of the aforementioned pathways to trigger an inflammatory response within the CNS (Kurimoto et al., 2010; Sun et al., 2011), it is hypothesized that GM-CSF is likely acting to suppress both PTEN and SOCS3 following injury, and in turn spurring an enhanced regenerative process that leads to robust axon regeneration at the lesion site. Sun and colleagues (2011) showed that sustained axon regeneration could be induced by deletion of both PTEN and SOCS3, and that although deletion of PTEN or SOCS3 alone can individually promote regeneration, this regeneration is often unsustained and ultimately reduced at 2 weeks post injury. This suggests that activation of both the mTOR and JAK/STAT signaling pathways may be required for sustained axon regeneration in the adult CNS. Interestingly, the mTOR signaling pathway has been shown to be active during development and is downregulated in adulthood (Liu et al., 2010). It is also further suppressed following injury, indicating that activation of the mTOR signaling pathway and enhancement of protein translation in the brain may be vital to axonal regeneration and the recovery of neural circuits following injury (Liu et al., 2010).

If GM-CSF is in fact up-regulating the activity of these pathways and inducing enhanced protein translation in the brain, it may very well have a role as a therapeutic agent following injury. This thesis attempts to examine the mechanistic role of GM-CSF in promoting axon growth and cell survival using a fully established RGC-primary cell culture system. By enhancing the phosphorylation of both STAT3 and S6, GM-CSF may not only be initiating axonal regeneration at the lesion site; it is also likely perpetuating it as time goes on. In addition to modulating the PTEN/mTOR and JAK/STAT signaling pathways, GM-CSF has also been shown to reduce glial scarring, attenuate CSPG 
expression at the lesion site, increase expression of neurotrophic growth factors, and facilitate phagocytosis of axonal and myelin debris following axonal degeneration (Huang et al., 2009; Ousman \& David, 2001). These combined effects imply a considerable role for GM-CSF in the treatment of traumatic brain and spinal cord injury, and in several neurodegenerative disorders including glaucoma, Parkinson's disease and Multiple Sclerosis.

\section{Determining GM-CSF's Role In CNS Regeneration}

It is often difficult to study CNS regeneration due to technical difficulties associated with the various assays involved (Newbern et al., 2009). Spinal cord injury has generally been the most commonly used model for regeneration, however replicating and standardizing these models is extremely difficult (Newbern et al., 2009). Additionally, if researchers can promote axonal growth, it is often difficult to trace regenerating axons over many spinal cord segments and to discriminate between axon regeneration and compensatory sprouting at the lesion site (Newbern et al., 2009). Because of these difficulties, lesioning the optic nerve has become a common model for studying CNS regeneration. It is much more easily controlled and allows for researchers to quantitatively assess strategies that might promote regeneration (Newbern et al., 2009). Culturing RGCs allows researchers to fully examine the effects of signaling molecules such as GM-CSF on both neuronal survival and axonal regeneration. It is also beneficial in that it allows for the examination of molecular mechanisms that may be involved in the effects that are observed. 


\section{Research Objectives and Hypotheses}

Several studies have now shown that GM-CSF activates a downstream signaling cascade that ultimately results in increased levels of neuronal survival both in vitro and in vivo (Bauer, Kerr, \& Patterson, 2007; Bouhy et al., 2006; Gu, Chiang, Zhu, Findley, \& Zhou, 2007; Kim et al., 2009; Mangano et al., 2011; Schallenberg, Charalambous \& Thanos, 2009). However, the intermediate effectors of this cascade have yet to be identified.

SOCS3 is of particular interest as it has been shown to mediate STAT3 levels via the JAK/STAT signaling pathway (Bareyre et al., 2011; Leibinger et al., 2009; Smith et al., 2009). Additionally, co-deletion of SOCS3 and PTEN has been shown to lead to robust regeneration in in vivo models of CNS injury (Sun et al., 2011). This suppression ultimately leads to the activation of the mTOR and JAK/STAT signaling pathways (Liu et al., 2010; Park et al., 2008; Sun et al., 2011). However, while co-deletion of these two signaling molecules appears to result in robust regeneration, individual deletion of either of these factors results in only moderate sprouting and regeneration (Sun et al., 2011).

As a result, it is hypothesized that GM-CSF administration results in enhanced axonal regeneration in vitro; and that GM-CSF is acting to not only increase expression of pSTAT3 by suppressing SOCS3, but to also increase expression of $\mathrm{pS} 6$ by suppressing PTEN. This hypothesis is based on previous studies that have shown that STAT3 predominantly acts to initiate axon regeneration as opposed to perpetuating it (Bareyre et al., 2011; Stosser et al., 2011). As a result, it can only be assumed that other signaling pathways and molecules are likely involved in GM-CSF's effect on CNS neurons. 
This thesis project examined the effects of GM-CSF administration on neuronal survival and axonal regeneration across different developmental time points. The effect of GM-CSF on CNS regeneration was tested using day 6 RGCs and day 10 RGCs in culture. The molecular underpinnings of these effects were also examined through the use of biochemical assays. In particular, pS6 and pSTAT3 were investigated as possible indicators of JAK/STAT and mTOR activity.

\section{Objectives.}

1. To determine the effect of GM-CSF on CNS regeneration by testing its effects on RGCs in culture across developmental time points.

2. To determine the signaling mechanisms underlying the GM-CSF-mediated effects on RGCs in primary tissue culture.

Hypothesis 1. If GM-CSF does in fact promote survival and axon growth, it is hypothesized that RGCs treated with GM-CSF will have significantly larger cell counts and significantly longer axonal projections than untreated RGCs in culture. A developmental decrease in the regenerative capacity of RGCs is also predicted when comparing results across different postnatal days.

Rationale. GM-CSF has been shown to induce compensatory sprouting around the lesion site and promote regeneration of damaged neuronal cell bodies in both in vitro and in vivo models of CNS injury (Bouhy et al., 2006; Huang et al., 2009; Mangano et al., 2011; Ousman \& David, 2001). It was therefore proposed that GM-CSF administration would also have positive effects on RGCs in culture. Specifically, if GM- 
CSF does promote axon growth, it could be hypothesized that RGCs treated with GMCSF would have significantly longer axonal projections than untreated RGCs in culture.

Hypothesis 2. It is hypothesized that GM-CSF treatment effects are due to activation of the JAK/STAT and/or mTOR signaling pathways. Through phosphorylation of both S6 and STAT3, protein translation is increased, resulting in an enhanced capacity for survival and regeneration (Kang et al., 2009; Kurimoto et al., 2010; Liu et al., 2010; Sun et al., 2011).

Rationale. Previous evidence indicates that genetic ablation of both PTEN and SOCS3 (negative regulators of mTOR and JAK/STAT signaling) in mice promotes prolonged and extensive axon regeneration in vivo (Park et al., 2008; Smith et al., 2009; Sun et al., 2011). If GM-CSF enhances axonal regeneration via a STAT3 dependent mechanism, then it is hypothesized that blocking STAT3 signaling prior to GM-CSF treatment would reduce the effects seen following GM-CSF administration. Similarly, if GM-CSF enhances axonal regeneration via an S6 dependent mechanism, it is hypothesized that blocking activity of the mTOR pathway prior to GM-CSF administration would also significantly reduce axonal regeneration of RGCs in culture. Individually blocking both JAK/STAT and mTOR signaling prior to GM-CSF administration would help to determine the extent that each of these pathways is involved in mediating the effects that are seen following GM-CSF treatment. Additionally, if deletion of both of these mechanisms completely ablates the effects that are seen following GM-CSF administration it can be assumed that the effects of GM-CSF 
administration are a result of activation of the JAK/STAT and mTOR signaling pathways.

Experiment. Retinal ganglion cell cultures were carried out using postnatal day 6 and postnatal day 10 mice. Each culture utilized retinas from 18 mice, with 3 mice from each litter assigned to one of 5 treatment groups: GM-CSF, GM-CSF + AG490 (an inhibitor of JAK/STAT signaling), GM-CSF + rapamycin (an inhibitor of mTOR signaling), GM-CSF + AG490 + rapamycin or control (Figure 2). RGCs from each treatment group were pooled prior to incubation and evenly distributed across 3 treatment wells, using a hemacytometer to confirm equal cell counts. Treatment groups were then compared using immunohistochemical assays to determine whether GM-CSF treatment resulted in larger cell counts and enhanced neuritic outgrowth, as well as to determine the presence of both pSTAT3 and pS6 in these groups. 


\section{CULTURE PLATE TREATMENT GROUPS}

L1
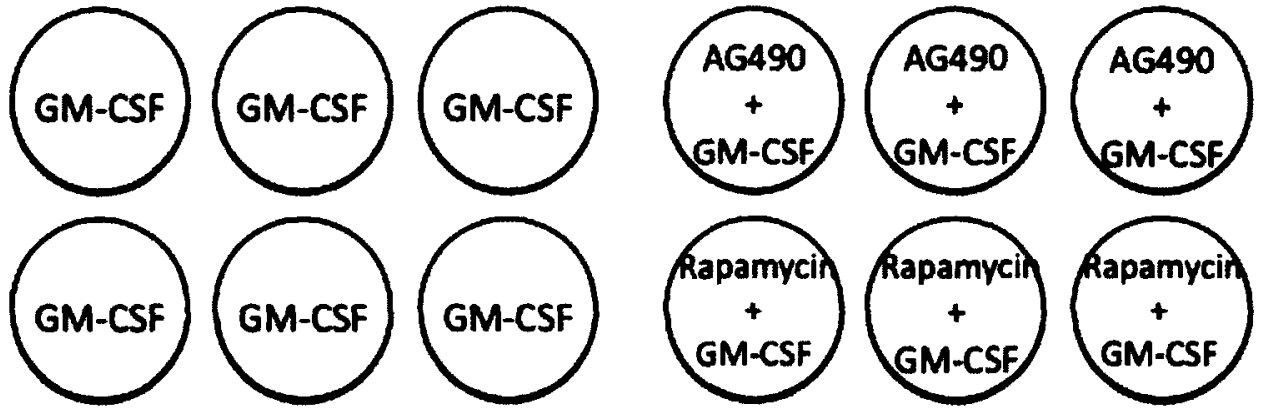

L2
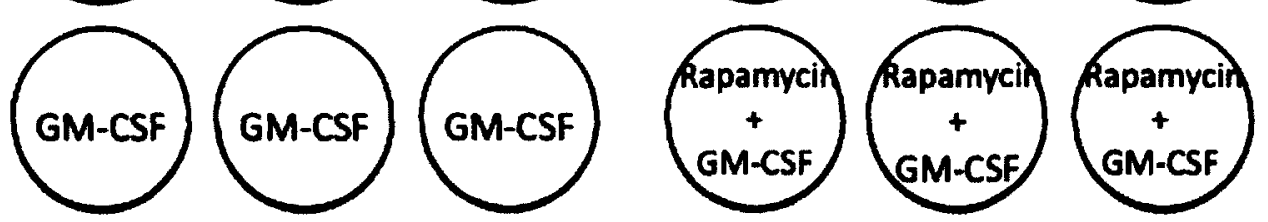

L3
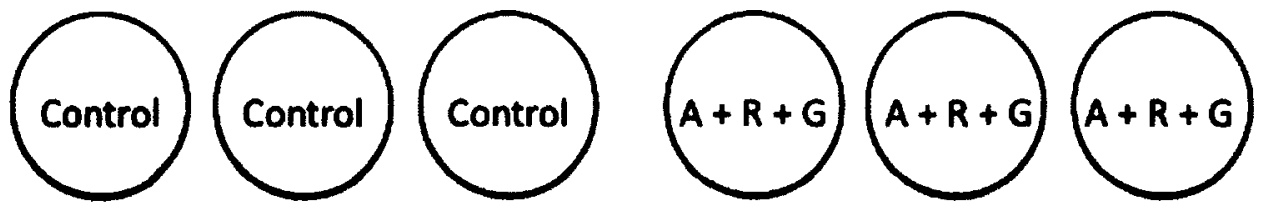

Figure 2. Culture Plate Treatment Group Design. Each culture contained RGCs from $18 \mathrm{C} 57$ black wild type mice. Each treatment group consisted of 3 animals taken from one of 3 litters (L1, L2 or L3). RGCs from these 3 animals were then pooled and assigned to one of 5 treatment groups: GM-CSF, control, AG490 + GM-CSF, rapamycin + GM-CSF or AG490 + rapamycin + GM-CSF $(A+R+G)$. These pooled samples were then treated immediately prior to plating and evenly distributed across 3 treatment wells. 


\section{METHODS \& PROCEDURES}

\section{Animals}

All experiments conformed to the guidelines of the Canadian Council on Animal Care and were carried out with the approval of the Carleton University Animal Care Committee. All experiments were performed on C57 black wild type mice.

\section{Isolation and Culture of Retinal Ganglion Cells}

Eyes of C57BL mice were isolated at postnatal days 6 and 10 and retinas were rapidly dissected and placed in a digestion solution containing papain and L-cysteine (Worthington), prior to incubation at $37^{\circ} \mathrm{C}$. Cells were then centrifuged for 5 minutes and solution was removed. Samples were diluted in final medium containing 1:1 concentrations of Dulbecco's Modified Eagle's Medium (DMEM) and Ham's F12 nutrient mixture, 10\% fetal bovine serum (FBS), $1 \% \mathrm{~N} 2$ and $1 \%$ penicillin/streptomycin. GM-CSF (20ng/ml) and/or AG490 (15 $\mu \mathrm{g} / \mathrm{ml})$ (Liu \& Snider, 2001) and/or rapamycin $(0.10 \mu \mathrm{g} / \mathrm{ml})$ (Tavazoie, Alvarez, Ridenour, Kwiatkowski \& Sabatini, 2005) were diluted in solution according to treatment group at the time of plating. Cell count was determined through the use of a hemacytomer and cells were plated equally across 18 wells coated with $20 \mu \mathrm{g} / \mathrm{ml}$ poly-D-lysine and $10 \mu \mathrm{g} / \mathrm{ml}$ laminin. RGCs were cultured for a total of 72 hours and cells were fixed in a $4 \%$ paraformaldehyde solution (Grozdanov, Muller, Sengottuvel, Leibinger, \& Fischer, 2010), prior to carrying out immunocytochemistry. 


\section{Immunocytochemistry}

Samples were treated with primary antibody solutions containing 1:1000 concentrations of $\beta$-tubulin mAb (Invitrogen Molecular Probes) and Anti-Ribosomal Protein pS6 Rabbit mAb, or $\beta$-tubulin mAb (1:1000) and pSTAT3 (1:500) Rabbit mAb (Invitrogen Molecular Probes). All antibodies were diluted in a solution of $0.01 \mathrm{M}$ PBS containing $0.5 \%$ Triton $\mathrm{X} 100$.

Following primary antibody treatment, samples were stored for 24 hours at $4^{\circ} \mathrm{C}$. Plates were then washed three times each for 10 minutes with $0.01 \mathrm{M}$ PBS and treated with secondary antibody solutions containing 1:200 concentrations of Alexa 488 donkey anti-rabbit IgG or Alexa 555 anti-mouse IgG. Plates were placed in the dark for one hour and then once again washed three times each for 10 minutes with $0.01 \mathrm{M}$ PBS, before being covered with $0.01 \mathrm{MPBS}$ and stored at $4^{\circ} \mathrm{C}$.

\section{Data Analysis}

Neurite length. Images were taken using a Zeiss Axiovert 40 inverted microscope and a $40 \mathrm{X}$ objective. Neurite length was manually quantified using SPOT Imaging Solutions software. Thirty neurite lengths from each treatment group were measured and an average for each treatment well was determined - only the longest neurites from each group were evaluated. A 2X5 ANOVA and Tukey's post hoc test was performed to compare both P6 and P10 developmental time points against treatment group (GM-CSF, GM-CSF + AG490, GM-CSF + rapamycin, GM-CSF + AG490 + rapamycin, and control). 
Survival. Nine images were taken across each well using a Zeiss Axiovert 40 inverted microscope and a 40X objective (Figure 3). The number of cells in each $240 \mathrm{X}$ $325 \mu \mathrm{m}$ frame was counted manually. Survival was assessed using a univariate ANOVA and Tukey's post hoc test to compare developmental time point (P6) and treatment group (GM-CSF, GM-CSF + AG490, GM-CSF + rapamycin, GM-CSF + AG490 + rapamycin, control). P10 data was not included in this analysis due to minimal survival outcomes.

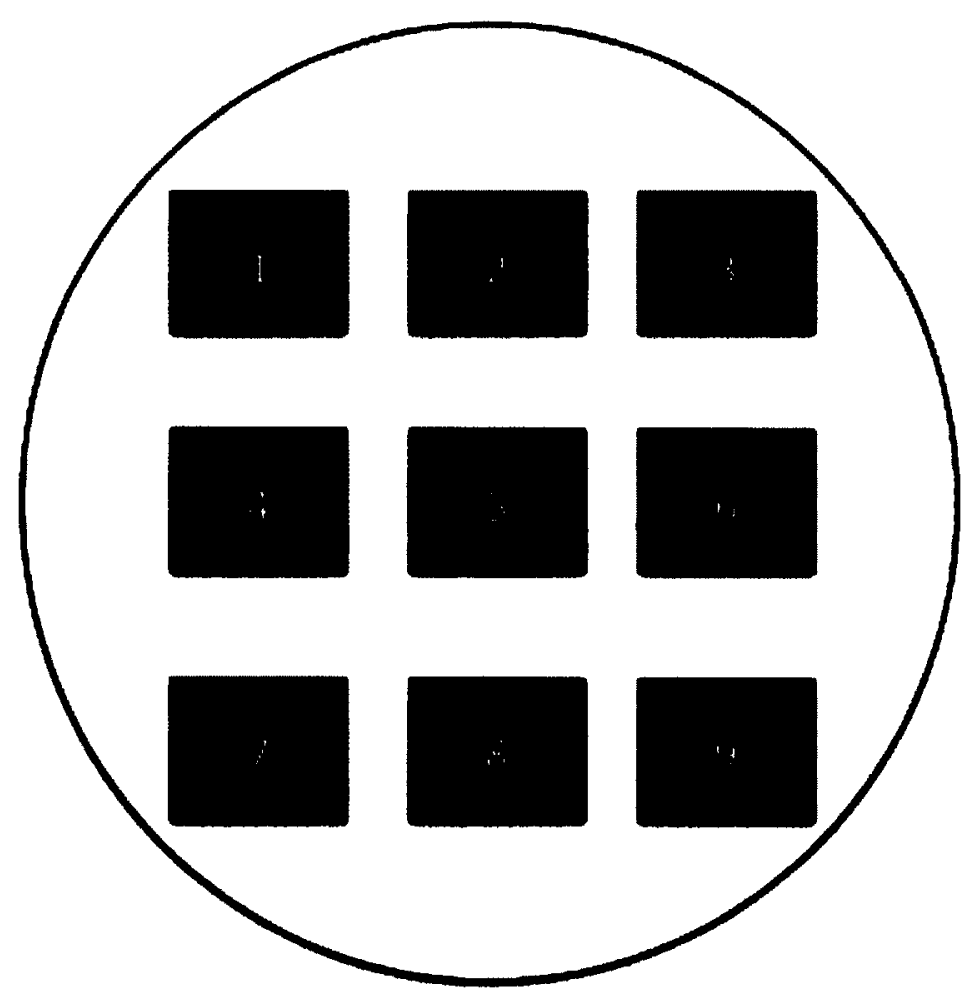

Figure 3. Survival Images Taken Across Each Well. Cells from each 240 X $325 \mu \mathrm{m}$ frame were manually counted and assessed to determine survival effects. 


\section{RESULTS}

\section{Axon Regeneration}

In order to assess the effects of GM-CSF administration on CNS axon growth at different developmental stages, a $2 \mathrm{X} 5$ analysis of variance (ANOVA) was conducted to determine if neurite length varied according to postnatal date ( $\mathrm{P} 6$ versus $\mathrm{P} 10$ ) and/or treatment group (GM-CSF versus control versus AG490 + GM-CSF versus rapamycin + GM-CSF versus AG490 + rapamycin + GM-CSF). According to Tukey's post hoc test, there was a main effect of postnatal day, $F(1,24)=325.40, p<.001, \eta 2 p=.96$, such that RGCs taken from postnatal day 6 wild type animals displayed longer neurite growth $(M=350.27, S D=70.45)$ compared to cells taken from wild type postnatal day 10 animals $(M=121.01, S D=59.18)$. A main effect of treatment group was also found, $F$ $(4,24)=21.01, p<.001, \eta 2 p=.86$, such that cells treated with GM-CSF $(M=295.81$, $S D=143.98)$ displayed neurite growth that was significantly longer than that of the control group $(M=213.89, S D=103.01), p<.01$, and cells treated with rapamycin and GM-CSF $(M=205.59, S D=164.52), p<.001$. Interestingly, cells treated with AG490 and GM-CSF $(M=259.73, S D=106.47)$, also showed neurite growth that was longer than that of the control group. Cells treated with AG490, rapamycin and GM-CSF ( $M=$ $143.02, S D=122.81$ ), displayed significantly shorter neurites when compared to each individual treatment group, $p<.05$, with the exception of the rapamycin and GM-CSF treatment group. Furthermore, these main effects were qualified by a significant interaction between postnatal day and treatment group, $F(4,24)=3.01, p<.05, \eta 2 p=$ .46. To better understand this interaction, simple effects analyses were performed to 
compare neurite length for each level of treatment group at both P6 and P10 time points (Table 1).

P6. As predicted, cells treated with GM-CSF showed enhanced axon growth when compared to controls, $p<.01$ (Figures 4 and 6). Surprisingly, neither the AG490 and GM-CSF nor the rapamycin and GM-CSF treated groups were significantly different from the GM-CSF treatment group. The double inhibitor group, treated with AG490, rapamycin and GM-CSF however, displayed significantly reduced neurite growth compared to all other treatment groups with the exception of controls, $p<.05$ (Figure 4).

P10. GM-CSF treated cells showed enhanced neurite growth when compared to both rapamycin and GM-CSF treated cells, $p<.05$, and cells treated with both inhibitors and GM-CSF, $p<.01$ (Figure 5). Interestingly, GM-CSF treated cells did not significantly differ from controls (Figure 7). However, cells treated with both inhibitors and GM-CSF displayed decreased neurite growth in relation to the AG490 and GM-CSF treatment group, $p<.01$. AG490 and GM-CSF treated RGC's showed significantly longer neurites than cells treated with rapamycin and GM-CSF, $p<.05$. 


\section{Table 1}

Mean Differences in Neurite Length $(\mu \mathrm{m})$ Expressed as a Function of Postnatal Date and Treatment Group

\begin{tabular}{|c|c|c|c|c|c|}
\hline \multirow[t]{2}{*}{ PD } & \multirow[t]{2}{*}{ Treatment Group } & & \multirow{2}{*}{$\begin{array}{c}\text { Mean } \\
\text { Difference }\end{array}$} & \multirow{2}{*}{$\begin{array}{c}\text { Standard } \\
\text { Error }\end{array}$} & \multirow[t]{2}{*}{ Sig. $\alpha$} \\
\hline & & & & & \\
\hline \multirow[t]{10}{*}{ P6 } & GM-CSF & $\mathrm{AG} 490+\mathrm{GM}-\mathrm{CSF}$ & 75.88 & 25.19 & .089 \\
\hline & & Rapamycin + GM-CSF & 80.94 & 25.19 & .061 \\
\hline & & AG490 + Rapamycin + GM-CSF & $179.86^{* * *}$ & 25.19 & .000 \\
\hline & & Control & $124.53^{* *}$ & 25.19 & .002 \\
\hline & AG490 + GM-CSF & Rapamycin + GM-CSF & 5.07 & 29.08 & 1.00 \\
\hline & & AG490 + Rapamycin + GM-CSF & $103.99 * *$ & 29.08 & .030 \\
\hline & & Control & 48.65 & 29.08 & .710 \\
\hline & Rapamycin + & AG490 + Rapamycin + GM-CSF & $98.92^{*}$ & 29.08 & .042 \\
\hline & & Control & 43.59 & 29.08 & .817 \\
\hline & $\begin{array}{l}\text { AG490 + Rapamycin } \\
+ \text { GM-CSF }\end{array}$ & Control & -55.34 & 29.08 & .555 \\
\hline \multirow[t]{10}{*}{ P10 } & GM-CSF & $\mathrm{AG} 490+\mathrm{GM}-\mathrm{CSF}$ & -3.73 & 25.19 & 1.00 \\
\hline & & Rapamycin + GM-CSF & $99.49^{*}$ & 25.19 & .014 \\
\hline & & AG490 + Rapamycin + GM-CSF & $125.71 * *$ & 25.19 & .002 \\
\hline & & Control & 39.30 & 25.19 & .781 \\
\hline & AG490 + & Rapamycin + GM-CSF & $103.22^{*}$ & 29.08 & .032 \\
\hline & & AG490 + Rapamycin + GM-CSF & $129.44^{* *}$ & 29.08 & .005 \\
\hline & & Control & 43.03 & 29.08 & .827 \\
\hline & $\begin{array}{l}\text { Rapamycin + } \\
\text { GM-CSF }\end{array}$ & AG490 + Rapamycin + GM-CSF & 26.22 & 29.08 & .992 \\
\hline & & Control & -60.19 & 29.08 & .447 \\
\hline & $\begin{array}{l}\text { AG490 + Rapamycin } \\
+ \\
\text { GM-CSF }\end{array}$ & Control & -86.41 & 29.08 & .097 \\
\hline
\end{tabular}




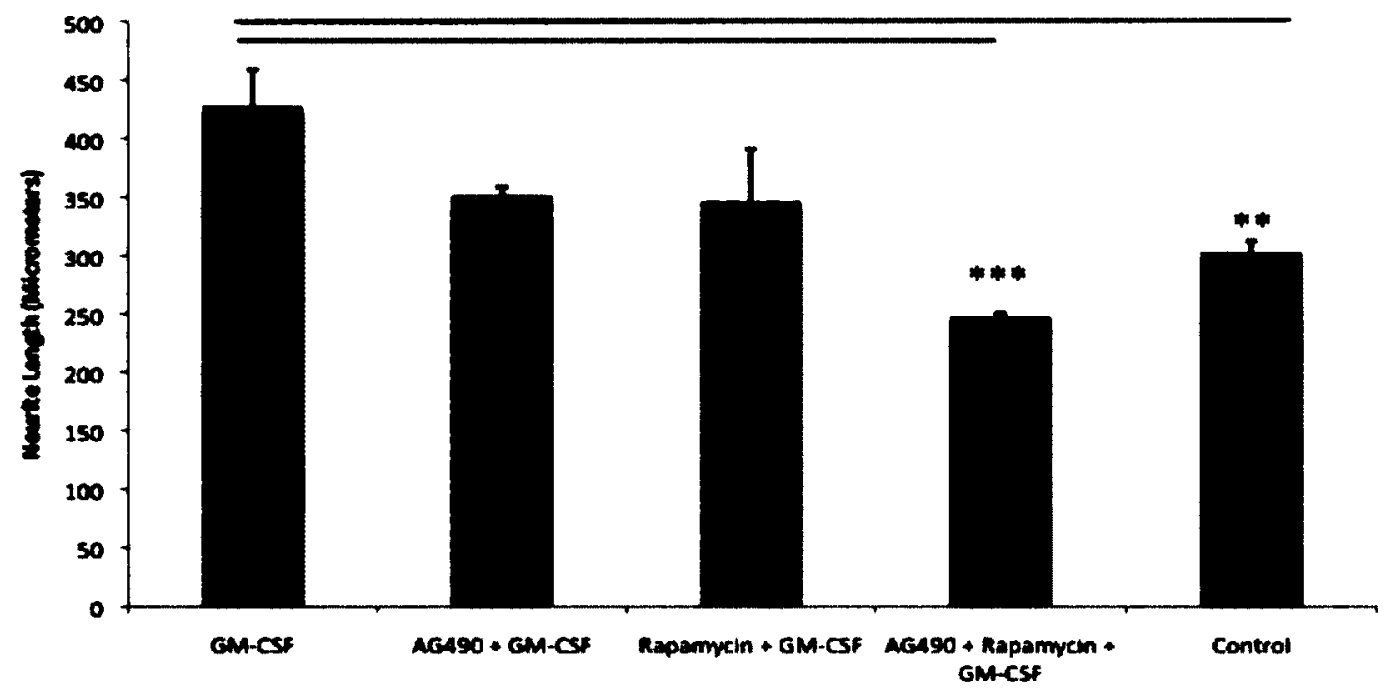

Figure 4. Mean Neurite Length of Postnatal Day 6 RGC's in Culture. Each mean is representative of the average neurite length $(\mu \mathrm{m})$ observed as a function of treatment group and postnatal day. Neurite length was assessed following a $72 \mathrm{~h}$ incubation period, and a 2X5 ANOVA and Tukey's post hoc test revealed that GM-CSF $(20 \mathrm{ng} / \mathrm{ml})$ treated neurons displayed neurite growth that was significantly longer than that of both controls and RGCs treated with AG490 $(15 \mu \mathrm{g} / \mathrm{ml})$ and rapamycin $(0.10 \mu \mathrm{g} / \mathrm{ml})$ in addition to GMCSF.

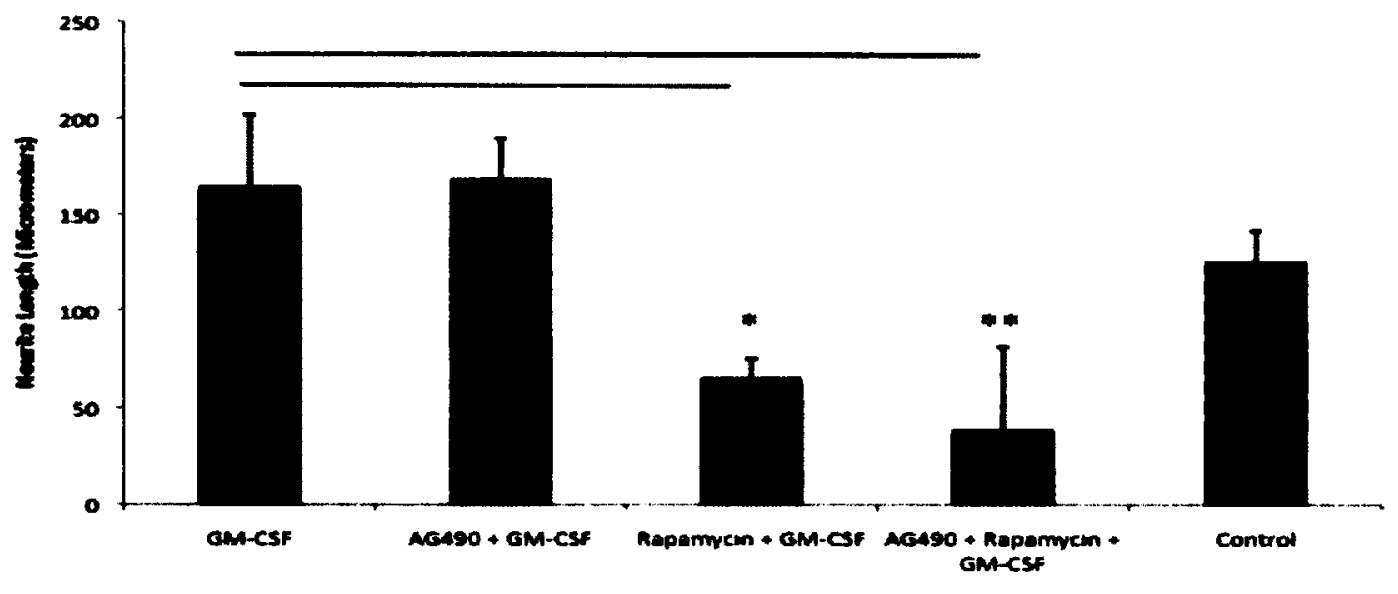

Figure 5. Mean Neurite Length of Postnatal Day 10 RGC's in Culture. Each mean is representative of the average neurite length $(\mu \mathrm{m})$ observed as a function of treatment group and postnatal day. Neurite length was assessed following a $72 \mathrm{~h}$ incubation period, and a 2 X5 ANOVA and Tukey's post hoc test revealed that GM-CSF (20ng/ml) treated neurons displayed neurite growth that was significantly longer than that of both rapamycin $(0.10 \mu \mathrm{g} / \mathrm{ml})$ treated RGCs, and RGCs treated with rapamycin, AG490 $(15 \mu \mathrm{g} / \mathrm{ml})$ and GM-CSF. 


\section{Postnatal Day 6}

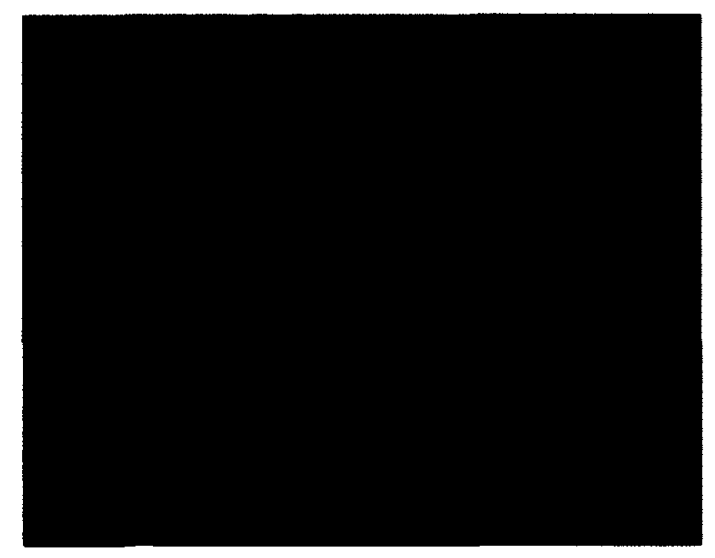

\section{Control}

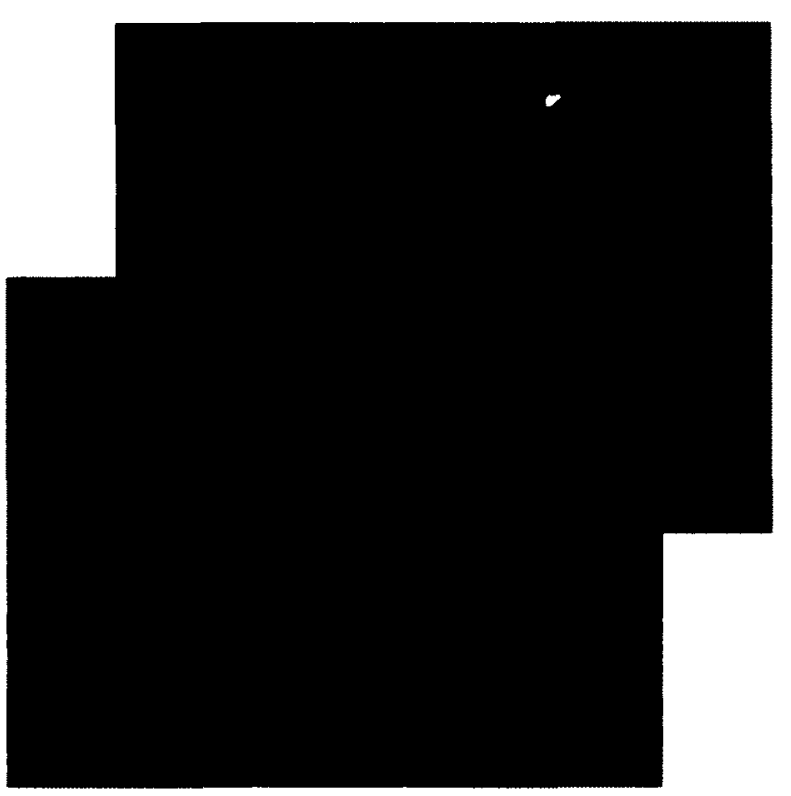

\section{GM-CSF}

Figure 6. Regeneration of Postnatal Day 6 RGCs in Culture. Cells shown here were cultured for $72 \mathrm{~h}$ in final medium containing varying concentrations of DMEM, F12, FBS, P15 and N2. GM-CSF treatment groups received a $20 \mathrm{ng} / \mathrm{ml}$ dose of GM-CSF immediately prior to plating. GM-CSF treated postnatal day 6 RGCs (stained here with $\beta$-tubulin) showed evidence of enhanced neurite growth in relation to controls. 


\section{Postnatal Day 10}

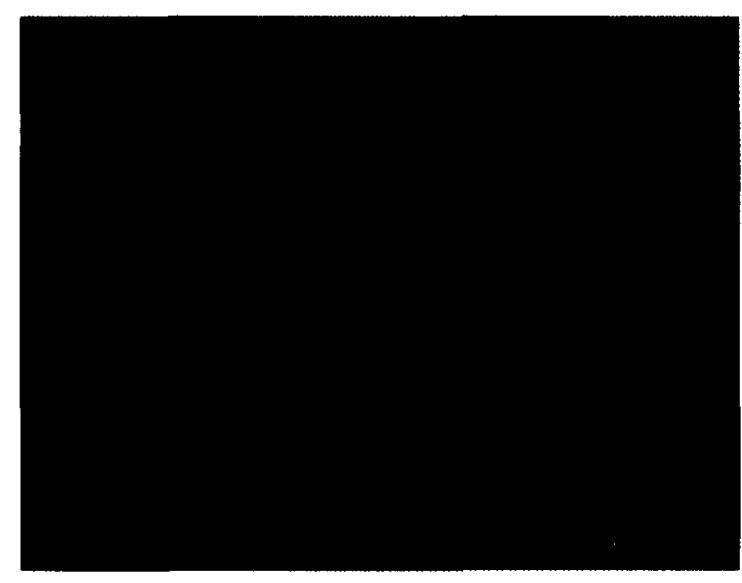

\section{GM-CSF}

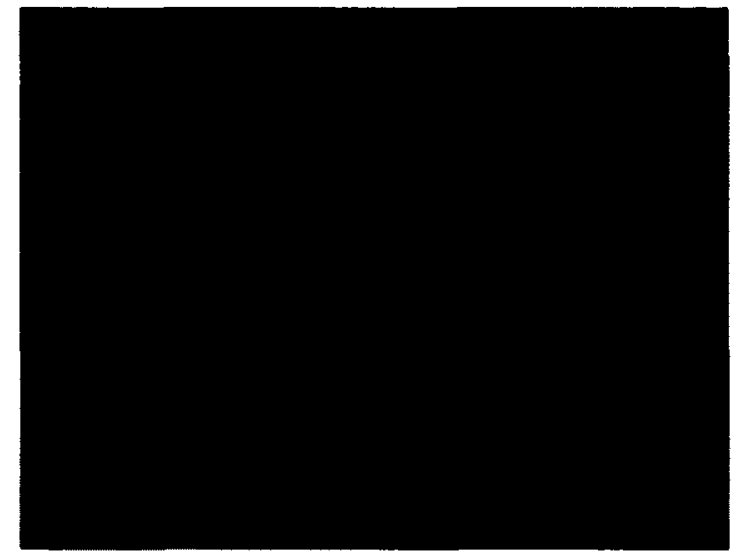

\section{Control}

Figure 7. Regeneration of Postnatal Day 10 RGCs in Culture. Cells shown here were cultured for $72 \mathrm{~h}$ in medium containing varying concentrations of DMEM, F12, FBS, P15 and N2. GM-CSF treatment groups received a $20 \mathrm{ng} / \mathrm{ml}$ dose of GM-CSF immediately prior to plating. While GM-CSF treated postnatal day 10 RGC's (stained here with $\beta$ tubulin) had a mean neurite length that was longer than that of controls, this difference did not reach significance, likely due to limited levels of survival in culture. 


\section{Survival}

P6. There was a main effect of treatment group, $F(4,18)=11.48, p<.001, \eta 2 p$ $=.78$. Tukey's post hoc test revealed that the GM-CSF treatment group $(M=84.70, S D$ $=10.87$ ) showed enhanced survival when compared to the Rapamycin and GM-CSF treatment group $(M=35.00, S D=3.46), p<.001$, and controls $(M=52.33, S D=13.32)$, $p<.05$, but not the AG490 treatment group $(M=97.67, S D=19.77)$ or the double inhibitor group ( $M=59.67, S D=18.15)$ (Table 2, Figures 8 and 9). Interestingly, the AG490 treatment group showed a similar trend, displaying a significantly larger cell count than the Rapamycin, $p<.001$, double inhibitor, $p<.05$, and control groups, $p<$ .01. 
Table 2

Mean Differences in Cell Count Expressed as a Function of Treatment Group

\begin{tabular}{|c|c|c|c|c|c|}
\hline \multirow[t]{2}{*}{ PD } & \multirow[t]{2}{*}{ Treatment Group } & & \multirow{2}{*}{$\begin{array}{c}\text { Mean } \\
\text { Difference }\end{array}$} & \multirow{2}{*}{$\begin{array}{c}\text { Standard } \\
\text { Error }\end{array}$} & \multirow[t]{2}{*}{ Sig. $\alpha$} \\
\hline & & & & & \\
\hline \multirow[t]{10}{*}{ P6 } & GM-CSF & AG490 + GM-CSF & -12.96 & 9.63 & .669 \\
\hline & & Rapamycin + GM-CSF & $49.70 * * *$ & 9.63 & .001 \\
\hline & & $\mathrm{AG} 490+$ Rapamycin + GM-CSF & 25.04 & 9.63 & .127 \\
\hline & & Control & $32.37^{*}$ & 9.63 & .034 \\
\hline & AG490+GM-CSF & Rapamycin + GM-CSF & $62.67^{* * *}$ & 11.11 & .001 \\
\hline & & $\mathrm{AG} 490+$ Rapamycin + GM-CSF & $38.00^{*}$ & 11.11 & .031 \\
\hline & & Control & $45.33^{* *}$ & 11.11 & .009 \\
\hline & Rapamycin + & AG490 + Rapamycin + GM-CSF & -24.67 & 11.11 & .232 \\
\hline & & Control & -17.33 & 11.11 & .545 \\
\hline & $\begin{array}{l}\text { AG490 + Rapamycin } \\
+ \text { GM-CSF }\end{array}$ & Control & 7.33 & 11.11 & .962 \\
\hline
\end{tabular}




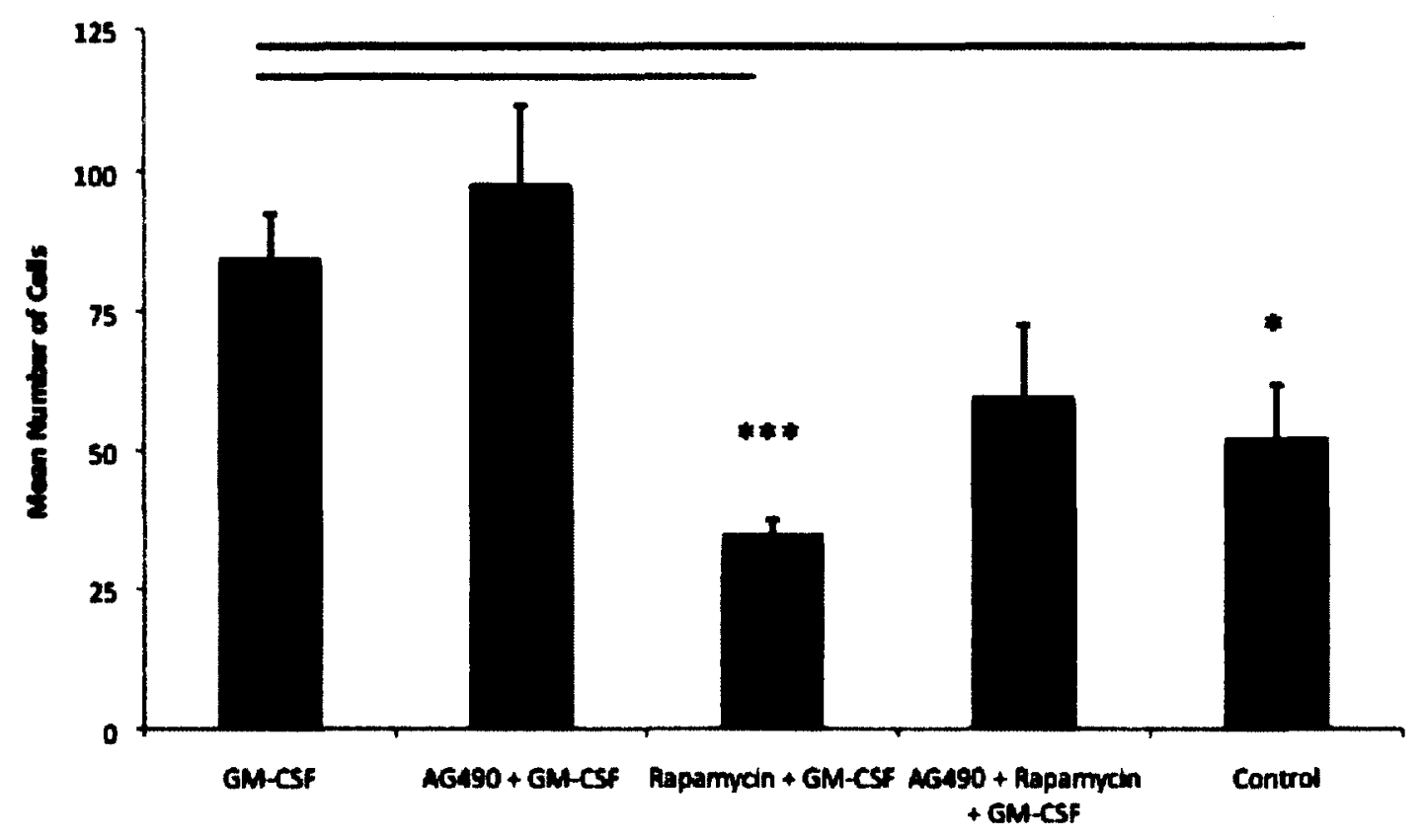

Figure 8. Mean Cell Count as an Indicator of Survival in Postnatal Day 6 RGCs. Cell counts are displayed as the average number of cells counted per $240 \times 325 \mu \mathrm{m}$ image per treatment well. Rapamycin $(0.10 \mu \mathrm{g} / \mathrm{ml})$ and GM-CSF $(20 \mathrm{ng} / \mathrm{ml})$ treated RGCs showed significantly lower levels of survival compared to both the GM-CSF and AG490 and GM-CSF treatment groups. The GM-CSF and AG490 and GM-CSF also showed enhanced survival when compared to controls. 


\section{Postnatal Day 6 Survival Effect}

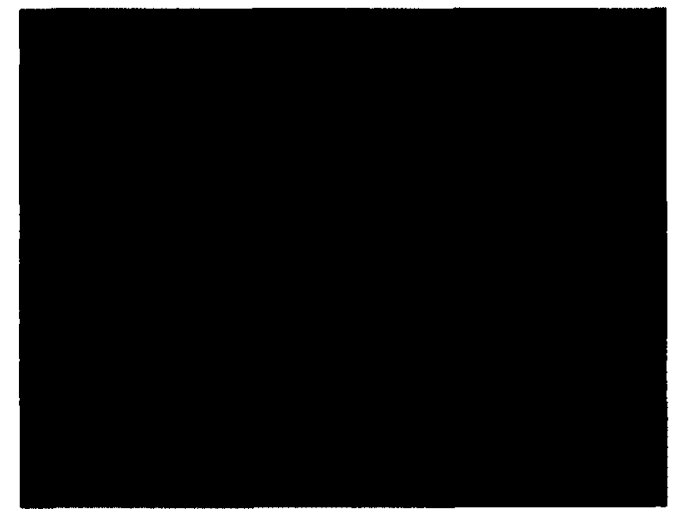

\section{Control}

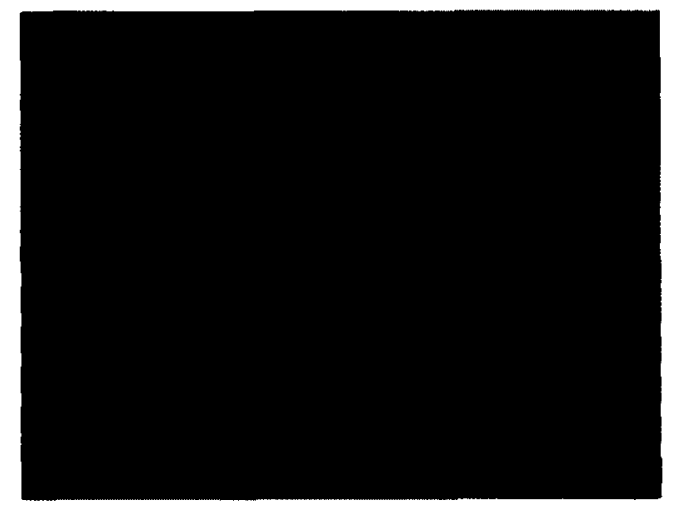

\section{GM-CSF}

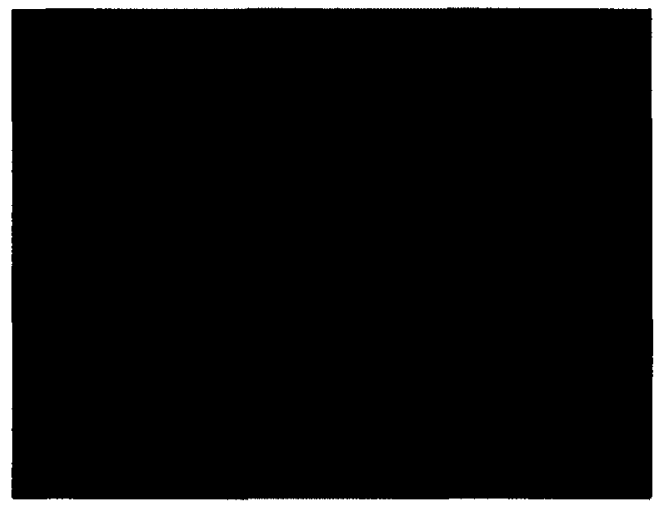

\section{Rapamycin \\ + GM-CSF}

Figure 9. Survival Effect in GM-CSF Treated Postnatal Day 6 RGCs. A significant survival effect was seen in GM-CSF $(20 \mathrm{ng} / \mathrm{ml})$ treated postnatal day 6 RGCs (stained here with $\beta$-tubulin) following a $72 \mathrm{~h}$ incubation period. Rapamycin $(0.10 \mu \mathrm{g} / \mathrm{ml})$ and GM-CSF $(20 \mathrm{ng} / \mathrm{ml})$ treated RGCs showed evidence of impaired survival, compared to that of both GM-CSF treated RGCs and controls. 


\section{Potential Mechanisms Mediating GM-CSF's Effects}

pS6. Positive pS6 immunostaining was observed in postnatal day 6, GM-CSF treated neurons (Figure 10). There was no evidence of any positive pS6 staining in any other treatment group at this postnatal date. Staining for pS6 in postnatal day 10 RGC's was inconclusive.

pSTAT3. There was very minimal evidence of pSTAT3 staining in GM-CSF treated postnatal day 6 and 10 RGC's. All other treatment groups at both postnatal dates showed no evidence of pSTAT3 staining. Although this result may be interpreted as a lack of pSTAT3 activity following GM-CSF administration, previous research suggests that minimal levels of phosphorylated STAT3 protein exist within RGCs, resulting in difficulty detecting this protein (Smith et al, 2009). 


\section{Postnatal Day 6 pS6 Immunostaining}
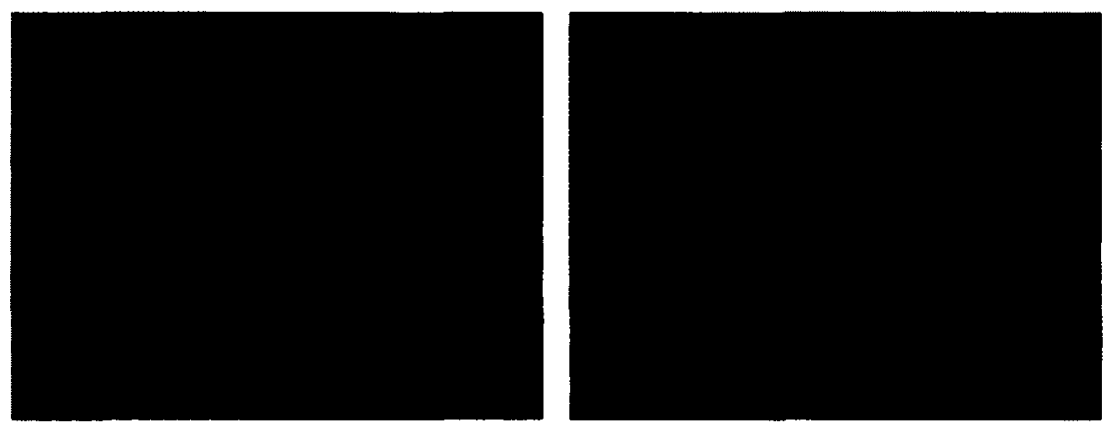

\section{Control}
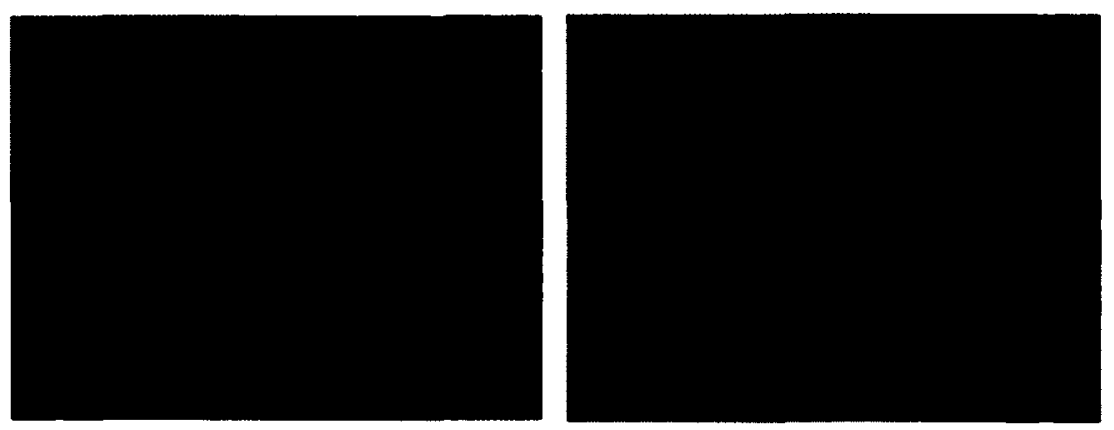

\section{GM-CSF}
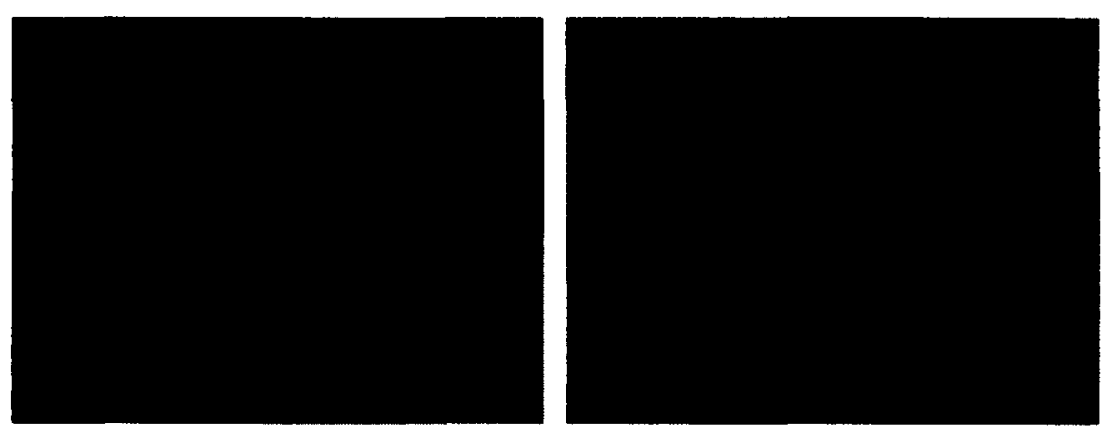

Rapamycin + GM-CSF

Figure 10. Immunostaining of pS6 in Postnatal Day 6 RGCs in Culture. Postnatal day 6 RGCs were fixed and stained with 1:1000 concentrations of $\beta$-tubulin $\mathrm{mAb}$ and pS6 mAb, following a $72 \mathrm{~h}$ incubation period. GM-CSF $(20 \mathrm{ng} / \mathrm{ml})$ treated RGCs showed positive pS6 staining compared to both negative controls. This indicates activity of the mTOR signaling pathway and enhanced protein translation in the GM-CSF mediated effects on regeneration. 


\section{DISCUSSION}

The hematopoietic cytokine, GM-CSF, has been shown to play a role in the proliferation and differentiation of a variety of cell types and is vital to triggering an immune response following injury (Hayashi et al., 2009; Kim et al., 2009; Manczak et al., 2009; Reddy et al., 2009; Qin et al., 2012). While GM-CSF exists primarily in the periphery, its ability to cross the blood-brain barrier and bind with endogenously occurring receptors within the CNS makes it a prime candidate for treating CNS injuries and illnesses (McLay et al., 1997; Ridwan et al., 2012). Following injury to the CNS, GM-CSF has been shown to mediate the recruitment of macrophages and microglia, while inhibiting gliosis and preserving the natural axonal arrangement and myelin structure following injury (Bouhy et al., 2006; Mangano et al., 2011; Ousman \& David, 2001; Reddy et al., 2009; Qin et al., 2012). It is also capable of reducing inhibition at the site of injury and promoting regenerative growth that is in some cases able to extend past the lesion site in order to reform connections that have been lost (Huang et al., 2009; Park et al., 2005; Yoon et al., 2007). Despite all of the evidence indicating a role for GM-CSF as a potential therapeutic agent for traumatic brain and spinal cord injury, stroke and a wide range of other neurodegenerative disorders, the molecular mechanisms underlying GM-CSF's actions are still unclear.

The objective of this study was to assess the regenerative effects of GM-CSF on CNS neurons over the course of development, while using an in vitro model to determine the molecular mechanisms involved. Following a $2 \times 5$ factorial analysis of our data using Tukey's post hoc test to compare axonal regeneration against postnatal date and treatment 
group, several conclusions can be made about the efficacy of GM-CSF's regenerative capabilities.

\section{Axonal Regeneration}

Recently, studies have shown evidence that GM-CSF has a positive effect on axon growth following injury, in in vivo models of stroke and spinal cord injury (Bouhy et al., 2006; Fathali, Lekic, Zhang, \& Tang, 2010; Kim et al., 2009; Mangano et al., 2011). Our in vitro model of regeneration supports these findings and further indicates a role for both the mTOR and JAK/STAT signaling pathways in the molecular underpinnings of the observed effects.

P6. Following a 72-hour incubation period, retinal ganglion cells extracted from postnatal day 6 , wild type animals, and treated with $20 \mathrm{ng} / \mathrm{ml}$ GM-CSF, showed enhanced neurite outgrowth in comparison to controls. This effect was also seen when comparing GM-CSF treated cells against RGCs treated with GM-CSF as well as AG490 and rapamycin, potent inhibitors of the JAK/STAT and mTOR signaling pathways. Furthermore, while individual suppression of each of these pathways resulted in axon lengths that were shorter than their unsuppressed counterparts, this difference failed to reach statistical significance, indicating that both pathways may be involved in the effects that were observed. This notion is further supported by data showing that both AG490/GM-CSF and rapamycin/GM-CSF treatment groups displayed mean axon lengths that were significantly longer than that of the double inhibitor group.

Interestingly, while both AG490 and rapamycin inhibitor groups demonstrated mean axon lengths that were longer than that of controls, these differences failed to reach 
significance. This suggests that individual inhibition of each of the aforementioned pathways may not be enough to cause a decrease in axon growth. One very likely possibility for this observed effect is that GM-CSF is targeting both the JAK/STAT and mTOR signaling pathways, and that upon individual suppression, each pathway is compensating for the other to produce regenerative effects. Another possibility is that the large population of cells plated may be having an alternate effect on growth via the release of neurotrophic factors. These processes could in turn mask some of the effects that were observed, resulting in a failure to reach significant levels.

P10. While GM-CSF signaling in P6 RGCs appears to incite JAK/STAT and mTOR signaling in order to affect regeneration, the data suggests that mTOR signaling may have a more prominent role in regeneration at later developmental time points. Remarkably, while P10 neurons treated with GM-CSF have a longer mean axon length than controls, this difference did not reach significance. This trend also occurred when looking at RGCs treated with both GM-CSF and AG490. Treatment with rapamycin and GM-CSF or GM-CSF, AG490 and rapamycin however, does appear to have a detrimental effect on regeneration, with each of these groups displaying shorter axon lengths than cells treated with GM-CSF alone. These results suggest that while both JAK/STAT and mTOR signaling are vital to axonal regeneration, the mTOR signaling pathway appears to play a larger role in these processes later on in development.

Given what is known about GM-CSF's effect on the regeneration of neurites in culture, it can be said that GM-CSF appears to enhance activity of both the JAK/STAT and mTOR signaling pathways to promote regeneration across developmental time 
points. Although GM-CSF treated P10 neurons had a mean neurite length that was longer than that of controls, this difference did not reach significance. This result can possibly be attributed to the fact that neurons from later developmental time points have a much greater difficulty surviving in culture than neurons from earlier developmental time points (Chen, Schneider, Martinou \& Tonegawa, 1997; Goldberg, Espinosa, Xu, Davidson, Kovacs, Barres, 2002). Chen \& colleagues (1997) in fact have shown that while embryonic neurons will readily extend neurites in culture, this active state of regeneration drops off significantly after postnatal day 4 . Following this postnatal date, regeneration is rarely seen in culture, likely due to a down-regulation of intrinsic growth factors. This suggests that the drop in survival capability that was observed across postnatal day 10 treatment groups may have been due to a developmental decrease in regenerative capacity, that is in turn compounded by culture conditions that are, by nature, unable to successfully support the survival of postnatal day RGCs. This may have had an impact on the observed postnatal day 10 results, and on these results subsequently failing to reach significance.

Another factor that must be considered is that $\mathrm{P} 10$ animals have more than 5 times fewer RGCs than P6 animals. This discrepancy in cell number is a natural part of developmental physiology that must be considered when plating cells in culture. In order to maintain developmental and physiological relevance, more cells were plated per well for P6 cultures than for P10 cultures. This difference in cell populations may contribute to the observed results, as the increased population of P6 cells may have allowed effects to be observed that were unable to be seen in the P10 due to a discrepancy in the number of surviving neurons. 


\section{Survival}

P6. Similar to what was seen with regard to regeneration, GM-CSF treated RGCs showed evidence of increased survival capability when compared to rapamycin and GMCSF treated neurons and controls. Interestingly, AG490 and GM-CSF treated RGCs displayed survival rates on par with that of GM-CSF treated RGC's. AG490 and GMCSF treated neurons had higher survival rates than Rapamycin and GM-CSF treated neurons, AG490, Rapamycin and GM-CSF treated neurons, and controls. This suggests that the mTOR signaling pathway is predominantly involved in promoting cell survival in P6 animals. Furthermore, this result suggests the likelihood of pS6 being a molecular target of GM-CSF. Phosphorylation of S6 rapidly increases levels of protein translation, and is a downstream target of the mTOR signaling pathway. If GM-CSF is in fact mediating its regenerative effects via an mTOR signaling cascade, it is possible that S6 is its ultimate target.

Another interesting factor that must be considered is that although GM-CSF treated RGCs exhibited enhanced survival when compared to the double inhibitor group, this difference did not appear to reach significance. One possibility for this result is that another signaling mechanism may be compensating for a lack of JAK/STAT and mTOR signaling in order to promote survival. One likely mechanism involves the initiation of the MAPK signaling pathway and activation of ERK, which has been shown to become activated following GM-CSF administration (Schallenberg, Charalambous \& Thanos, 2009), and also has the ability to indirectly activate mTOR and trigger phosphorylation of S6. 
P10. Due to the fact that there was minimal survival in all P10 treatment groups, precise survival rates could not be assessed at this developmental time point. However, it can be suggested that a lack of cellular survival is the main proponent affecting the lack of regeneration that is seen at later time points, and that GM-CSF may be attempting to counteract this lack of survival by targeting the mTOR signaling pathway. Given the fact that several in vivo studies have observed enhanced cellular survival due to GM-CSF administration in adult mice and rats (Mangano, et al., 2011, Huang, et al., 2009), this lack of survival in vitro is likely due to technical issues with culturing mature RGCs, as opposed to a lack of efficacy in relation to GM-CSF administration.

\section{Signaling Mechanisms}

It is clear that both JAK/STAT and mTOR signaling are involved in GM-CSF's effect on regeneration. However, the role of each of these pathways in the regenerative process must be further examined in order to qualify the possible mechanisms underlying GM-CSF activation in CNS neurons.

JAK/STAT signaling. It is well established that the JAK/STAT signaling pathway is important to CNS growth and regeneration. STAT3, a regulator of gene transcription, in particular has been shown to be rapidly phosphorylated following CNS injury (Bareyre et al., 2011; Stosser et al., 2011). Increased levels of STAT3 expression and phosphorylation are not only associated with initiating axonal regeneration, but also with synaptic remodeling in the brain and spinal cord (Bareyre et al., 2011; Stosser et al., 
2011). According to the data analysis however, AG490, a potent inhibitor of the JAK2 component of the JAK/STAT signaling pathway and an inhibitor of STAT3 production, does not prevent regeneration of neurites following GM-CSF administration. As previously mentioned, while RGCs treated with AG490, Rapamycin and GM-CSF did show diminished rates of survival and regeneration at the P6 time point, individual administration of either AG490 or Rapamycin in addition to GM-CSF was not enough to prevent regeneration of neurites in culture. This suggests that while STAT3 is typically rapidly phosphorylated following GM-CSF administration, inhibition of the JAK/STAT signaling pathway and suppression of STAT3 phosphorylation is not enough to prevent axonal regeneration from occurring. This result was further compounded by the fact that minimal pSTAT3 staining was found within GM-CSF treatment groups at either postnatal dates, suggesting that while some pSTAT3 activity is possible, it is unlikely that STAT3 is a main molecular target of GM-CSF.

Another factor to consider is that cells treated with AG490 and GM-CSF at the P10 developmental time point showed growth that was on par with their GM-CSF treated counterparts. This indicates that the role of STAT3 in regeneration may be further limited at later developmental time points, or that other signaling pathways may take over to compensate for a lack of STAT3 activation. These pathways may or may not be involved at earlier developmental time points. Interestingly, AG490 was also shown to have little effect on survival at the P6 time point, further indicating that STAT3 activation is typically associated with initiating axon growth as opposed to promoting survival (Bareyre et al., 2011). 
mTOR signaling. The mTOR signaling pathway is a strong promoter of CNS growth during development. As the individual reaches adulthood however, its activity is down-regulated and is, unfortunately, further suppressed following injury later in development (Liu et al., 2010). Given the fact that the mTOR signaling pathway has been shown to produce robust regeneration when activated following injury (Sun et al., 2011), the objective of this thesis was to determine whether GM-CSF might be targeting this pathway to produce regeneration in vitro. As previously mentioned, while RGCs treated with rapamycin, an inhibitor of mTOR signaling, AG490, an inhibitor of JAK/STAT signaling, and GM-CSF did show diminished rates of regeneration at the P6 time point, individual administration of either AG490 or rapamycin in addition to GMCSF was not enough to prevent regeneration of neurites in culture. Rapamycin and GMCSF treated RGCs however, were shown to exhibit significantly reduced survival capabilities when compared to their GM-CSF treated counterparts. Additionally, the rapamycin treated group was shown to display fewer cells on average than the P6 control group, although this result was not significant. This suggests a critical role for the mTOR signaling pathway following GM-CSF activation. Furthermore, rapamycin treated cells at the P10 developmental time point were shown to exhibit significantly impaired regenerative capabilities in relation to other treatment groups, further signifying that the mTOR signaling pathway and its molecular counterpart, S6, may be key targets of GMCSF signaling. 
In order to confirm this hypothesis, treatment groups were stained for $\mathrm{pS} 6$. As previously indicated, GM-CSF treated RGCs showed evidence of positive pS6 staining in comparison to rapamycin treated negative controls, verifying that phosphorylation of S6 is substantially up-regulated following treatment with GM-CSF (Figure 12).

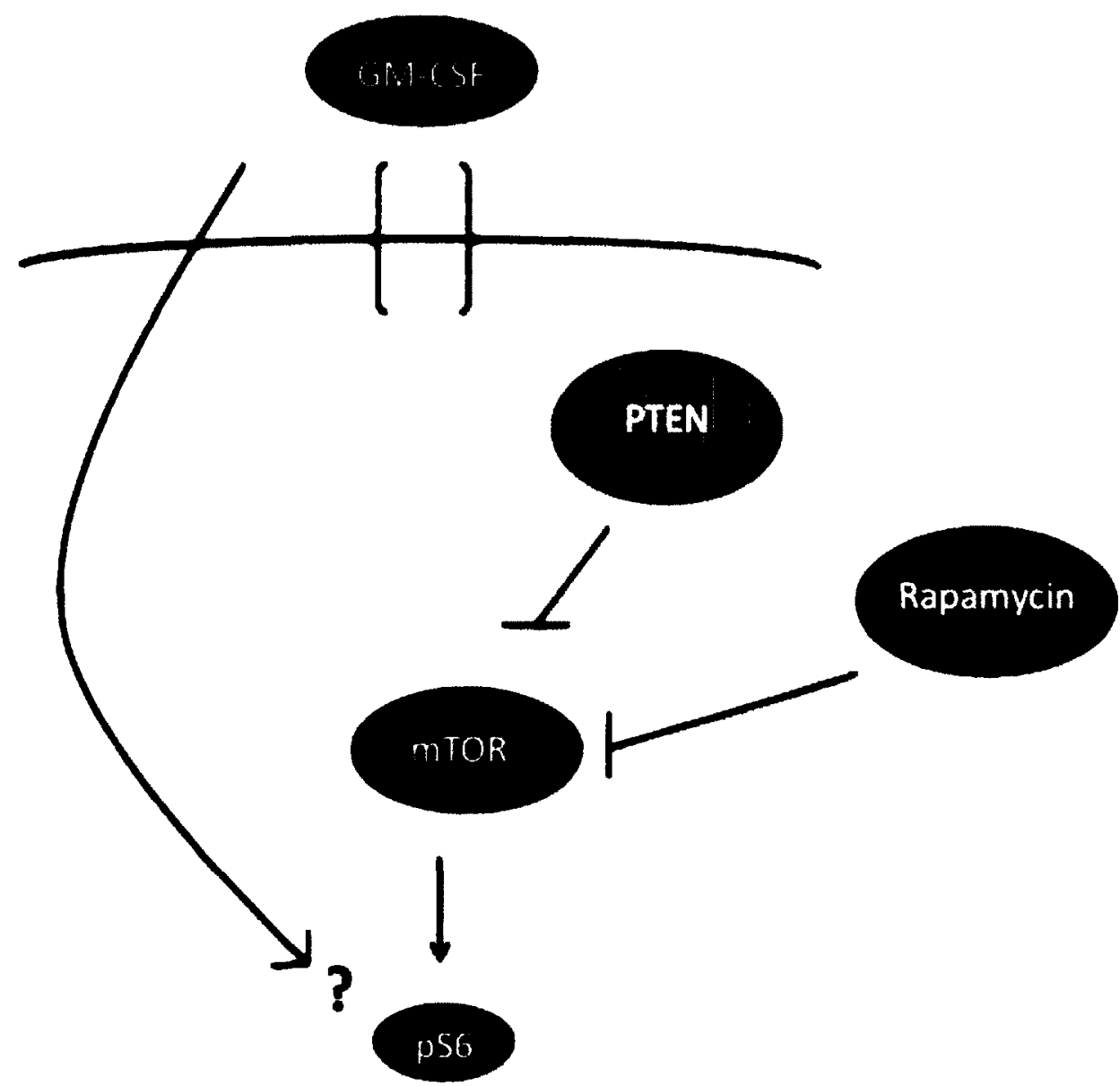

Figure 12. mTOR Signaling Mechanism. Deletion of PTEN has been shown to indirectly up-regulate activity of the mTOR signaling pathway to ultimately facilitate phosphorylation of the ribosomal protein S6. Rapamycin inhibits mTOR activity, suppressing phosphorylation of S6. GM-CSF is a potential mediator of this pathway and may act to increase mTOR activity and assist in regeneration following injury. 
SOCS3 \& PTEN. Sun and colleagues (2011), recently reported evidence of robust axon regeneration following co-deletion of both SOCS3, a negative regulator of the JAK/STAT signaling pathway, and PTEN, a negative regulator of the mTOR signaling pathway. They also suggested that while both of these pathways are heavily involved in mediating the injury response and in promoting regeneration, they also operate independently of one another (Sun et al., 2011). The current study however indicates that this may not be the case. While doubly inhibiting JAK/STAT and mTOR signaling dramatically decreased the regenerative capability of GM-CSF treated postnatal day 6 RGC's in culture, individual suppression of each of these pathways was not enough to significantly reduce regeneration of neurites. This indicates that there may be crosstalk between these pathways in order to promote regeneration when one is inhibited.

However, given that suppression of both of the aforementioned pathways produced significantly reduced neurite growth following GM-CSF treatment at both postnatal dates, and both double inhibitor groups showed levels of growth that were comparable to that of controls, this further emphasizes the roles of these pathways in regeneration, and perhaps suggests a role for PTEN and SOCS3 in GM-CSF mediated signaling effects. While it was not tested whether or not GM-CSF directly targets either of these molecular regulators, our data indicate that mTOR and its signaling counterpart pS6 are both activated following GM-CSF administration. Given that PTEN is a negative regulator of the mTOR signaling pathway, and that suppression of PTEN results in robust axon regeneration (Sun et al., 2011), it can be claimed that PTEN is a likely target of GM-CSF, and that GM-CSF may ultimately act to inhibit PTEN activity in order to up- 
regulate mTOR signaling and rapidly increase phosphorylation of the protein transcription factor $\mathbf{S 6}$.

SOCS3 on the other hand, is a negative regulator of the JAK/STAT signaling pathway. While AG490 triggered inhibition of JAK2/STAT3 signaling is not enough to abolish regenerative effects in P6 cultured RGC's, it does appear to have a synergistic effect when used in combination with rapamycin. This indicates that while suppression of JAK2/STAT3 signaling plays a role in reducing the regenerative capacity of CNS neurons following GM-CSF administration, it is not a main target of GM-CSF. This result is corroborated by data showing only minimal pSTAT3 staining among GM-CSF treatment groups, although this lack of staining may also be attributed to low levels of this particular protein within postnatal day RGCs. As a result, it would appear that SOCS3 is not a likely target of GM-CSF, despite it's well established role in regeneration.

\section{Conclusions \& Future Directions}

GM-CSF not only has the ability to reduce glial scarring and attenuate inhibition at the lesion site, it also has the ability to increase expression of neurotrophic growth factors and facilitate phagocytosis of axonal and myelin debris following injury (Bouhy et al., 2006; Mangano et al., 2011; Ousman \& David, 2001). GM-CSF is able to activate signaling pathways that have the ability to promote survival and regeneration of damaged cells and fibers following injury and can easily cross the blood brain barrier to react with endogenous receptors within the CNS (McLay et al., 1997). All of these factors make 
GM-CSF an excellent candidate to be a therapeutic agent for brain and spinal cord injury, stroke and a number of debilitating neurodegenerative disorders.

The purpose of this study was to use an in vitro model of the optic nerve to evaluate GM-CSF's regenerative effects, and to determine the possible mechanisms underlying GM-CSF's actions. It would appear that GM-CSF may not only promote extensive axon growth, but cellular survival as well. Results indicate that GM-CSF promotes regeneration via activation of the JAK/STAT and mTOR signaling pathways, and that pS6 is a key molecular target of this signaling cascade. As a result, it can be suggested that PTEN, a negative regulator of mTOR signaling and possibly S6 phosphorylation, may also be a target of GM-CSF, although further experiments will be required to confirm this.

Although there appears to be some cross-talk occurring between the JAK/STAT and mTOR signaling pathways following GM-CSF administration in postnatal day 6 animals in order to produce regenerative effects in vitro, pSTAT3 did not appear to play a significant role in the regenerative effects that were observed within this study. This is particularly interesting, especially given the fact that STAT3 has previously been shown to be rapidly phosphorylated following CNS injury (Bareyre et al., 2011; Smith et ak., 2009; Stosser et al., 2011). Nevertheless this result suggests that while JAK/STAT signaling may be up-regulated following GM-CSF administration, pSTAT3 is not a main molecular target of GM-CSF.

While it is possible that other downstream effectors of the JAK/STAT signaling pathway may be involved in GM-CSF's regenerative effects, the data strongly suggest that both the postnatal day 6 survival effects and postnatal day 10 regenerative effects 
that have been observed are predominantly mTOR mediated. As a result, it can be concluded that SOCS3 is a possible, but unlikely target of GM-CSF.

While GM-CSF appears to act predominantly via JAK/STAT and mTOR dependent mechanisms to promote the phosphorylation of downstream target proteins, it is quite possible that other signaling pathways may also be involved. Several studies for example have confirmed GM-CSF's ability to activate Bcl-2, an anti-apoptotic molecule, in order to promote survival (Chen et al., 1997; Goldberg et al., 2002). A recent study carried out by Schallenberg and colleagues (2009), also showed evidence that GM-CSF actively regulates ERK1/2 pathways to prevent induced cell death. While we have elucidated what appear to be the main mechanisms underlying GM-CSF activation following injury, further research will be required in order to clarify all of the mechanisms that contribute to GM-CSF's many effects on the CNS. Fully understanding the mechanisms underlying the effects that are observed following GM-CSF treatment will help shed light on possible new therapeutic approaches to treating traumatic brain and spinal cord injury, glaucoma, stroke, and a host of other debilitating neurodegenerative diseases. 


\section{REFERENCES}

Bareyre, F. M., Garzorz, N., Lang, C., Misgeld, T., Buning, H., \& Kerschensteiner, M. (2011). In vivo imaging reveals a phase-specific role of STAT3 during central and peripheral nervous system axon regeneration. Proc Natl Acad Sci US A, 108(15), 6282-6287. doi: 10.1073/pnas.1015239108

Bauer, S., Kerr, B. J., \& Patterson, P. H. (2007). The neuropoietic cytokine family in development, plasticity, disease and injury. Nat Rev Neurosci, 8(3), 221-232. doi: $10.1038 / \mathrm{nrn} 2054$

Benowitz, L. I., \& Yin, Y. (2010). Optic nerve regeneration. [Research Support, N.I.H., Arch Ophthalmol, 128(8), 1059-1064. doi: 10.1001/archophthalmol.2010.152

Bouhy, D., Malgrange, B., Multon, S., Poirrier, A. L., Scholtes, F., Schoenen, J., \& Franzen, R. (2006). Delayed GM-CSF treatment stimulates axonal regeneration and functional recovery in paraplegic rats via an increased BDNF expression by endogenous macrophages. FASEB J, 20(8), 1239-1241. doi: 10.1096/fj.05$4382 \mathrm{fje}$

Buffo, A., Rolando, C., \& Ceruti, S. (2010). Astrocytes in the damaged brain: molecular and cellular insights into their reactive response and healing potential. Biochem Pharmacol, 79(2), 77-89. doi: 10.1016/j.bcp.2009.09.014

Chen, D.F., Schneider, G.E., Martinou, J.C. \& Tonegawa, S. (1997). Bcl-2 promotes regeneration of severed axons in mammalian CNS. Nature, 385(6615), 434-439.

Coleman, M. (2005). Axon degeneration mechanisms: commonality amid diversity. Nat Rev Neurosci, 6(11), 889-898. doi: 10.1038/nrn1788 
David, S., \& Aguayo, A. J. (1981). Axonal elongation into peripheral nervous system "bridges" after central nervous system injury in adult rats. Science, 214(4523), 931-933.

Fathali, N., Lekic, T., Zhang, J. H., \& Tang, J. (2010). Long-term evaluation of granulocyte-colony stimulating factor on hypoxic-ischemic brain damage in infant rats. Intensive Care Med, 36(9), 1602-1608. doi: 10.1007/s00134-010-1913-6

Fischer, D. (2010). What are the principal mediators of optic nerve regeneration after inflammatory stimulation in the eye? Proc Natl Acad Sci U S A, 107(3), E8; author reply E9. doi: 10.1073/pnas.0912942107

Fitch, M. T., \& Silver, J. (2008). CNS injury, glial scars, and inflammation: Inhibitory extracellular matrices and regeneration failure. Exp Neurol, 209(2), 294-301.

Gaudet, A. D., Popovich, P. G., \& Ramer, M. S. (2011). Wallerian degeneration: gaining perspective on inflammatory events after peripheral nerve injury. $J$ Neuroinflammation, 8, 110. doi: 10.1186/1742-2094-8-110

Goodwin, C., Yang, Z., Yin, F., Yu, M. \& Chan, R. (2012). Genetic disruption of the PI3K regulatory subunits, $\mathrm{p} 85 \alpha, \mathrm{p} 55 \alpha$, and $\mathrm{p} 50 \alpha$ normalizes mutant PTPN11induced hypersensitivity to GM-CSF. Haematologica, 97(7), 1042-1047.

Goldberg, J.L., Espinosa, J.S, Xu, Y., Davidson, N., Kovacs, G. \& Barres, B.A. (2002). Retinal ganglion cells do not extend axons by default: Promotion by neurotrophic signaling and electrical activity. Neuron, 33, 689-702.

Grozdanov, V., Muller, A., Sengottuvel, V., Leibinger, M., \& Fischer, D. (2010). A method for preparing primary retinal cell cultures for evaluating the neuroprotective and neuritogenic effect of factors on axotomized mature CNS 
neurons. Curr Protoc Neurosci, Chapter 3, Unit3 22. doi: 10.1002/0471142301.ns0322s53

Gu, L., Chiang, K. Y., Zhu, N., Findley, H. W., \& Zhou, M. (2007). Contribution of STAT3 to the activation of survivin by GM-CSF in CD34+ cell lines. Exp Hematol, 35(6), 957-966. doi: 10.1016/j.exphem.2007.03.007

Ha, Y., Park, H. S., Park, C. W., Yoon, S. H., Park, S. R., Hyun, D. K., . . Park, H. C. (2005). Synthes Award for Resident Research on Spinal Cord and Spinal Column Injury: granulocyte macrophage colony stimulating factor (GM-CSF) prevents apoptosis and improves functional outcome in experimental spinal cord contusion injury. Clin Neurosurg, 52, 341-347.

Hayashi, K., Ohta, S., Kawakami, Y., \& Toda, M. (2009). Activation of dendritic-like cells and neural stem/progenitor cells in injured spinal cord by GM-CSF. Neurosci Res, 64(1), 96-103. doi: 10.1016/j.neures.2009.01.018

Huang, X., Kim, J. M., Kong, T. H., Park, S. R., Ha, Y., Kim, M. H., . . Choi, B. H. (2009). GM-CSF inhibits glial scar formation and shows long-term protective effect after spinal cord injury. $J$ Neurol Sci, 277(1-2), 87-97. doi: 10.1016/j.jns.2008.10.022

Huebner, E. A., \& Strittmatter, S. M. (2009). Axon regeneration in the peripheral and central nervous systems. Results Probl Cell Differ, 48, 339-351. doi: 10.1007/400_2009_19

Hur, E. M., Yang, I. H., Kim, D. H., Byun, J., Saijilafu, Xu, W. L., . . Zhou, F. Q. (2011). Engineering neuronal growth cones to promote axon regeneration over 
inhibitory molecules. Proc Natl Acad Sci U S A, 108(12), 5057-5062. doi: 10.1073/pnas. 1011258108

Kang, K. H., Lemke, G., \& Kim, J. W. (2009). The PI3K-PTEN tug-of-war, oxidative stress and retinal degeneration. Trends Mol Med, 15(5), 191-198. doi: 10.1016/j.molmed.2009.03.005

Kim, H.J., Oh, J.S., An, S.S., Pennant, W.A., Gwak, S.J., Kim, A.N, Han, P.K, et al. (2012). Hypoxia-specific GM-CSF-overexpressing neural stem cells improve graft survival and functional recovery in spinal cord injury. Gene Therapy, 19(5), 513-521.

Kim, N. K., Choi, B. H., Huang, X., Snyder, B. J., Bukhari, S., Kong, T. H., . . Ha, Y. (2009). Granulocyte-macrophage colony-stimulating factor promotes survival of dopaminergic neurons in the 1-methyl-4-phenyl-1,2,3,6-tetrahydropyridineinduced murine Parkinson's disease model. Eur J Neurosci, 29(5), 891-900. doi: $10.1111 / j .1460-9568.2009 .06653 . x$

Kurimoto, T., Yin, Y., Omura, K., Gilbert, H. Y., Kim, D., Cen, L. P., . . Benowitz, L. I. (2010). Long-distance axon regeneration in the mature optic nerve: contributions of oncomodulin, cAMP, and pten gene deletion. $J$ Neurosci, 30(46), 1565415663. doi: $10.1523 /$ JNEUROSCI.4340-10.2010

Lee, J. K., Chow, R., Xie, F., Chow, S. Y., Tolentino, K. E., \& Zheng, B. (2010). Combined genetic attenuation of myelin and semaphorin-mediated growth inhibition is insufficient to promote serotonergic axon regeneration. $J$ Neurosci, 30(32), 10899-10904. doi: 10.1523/JNEUROSCI.2269-10.2010 
Lee, J. K., Geoffroy, C. G., Chan, A. F., Tolentino, K. E., Crawford, M. J., Leal, M. A., . .. Zheng, B. (2010). Assessing spinal axon regeneration and sprouting in Nogo-, MAG-, and OMgp-deficient mice. Neuron, 66(5), 663-670. doi: $10.1016 / \mathrm{j}$.neuron.2010.05.002

Leibinger, M., Muller, A., Andreadaki, A., Hauk, T. G., Kirsch, M., \& Fischer, D. (2009). Neuroprotective and axon growth-promoting effects following inflammatory stimulation on mature retinal ganglion cells in mice depend on ciliary neurotrophic factor and leukemia inhibitory factor. $J$ Neurosci, 29(45), 14334-14341. doi: 10.1523/JNEUROSCI.2770-09.2009

Lim, P. A., \& Tow, A. M. (2007). Recovery and regeneration after spinal cord injury: a review and summary of recent literature. [Review]. Ann Acad Med Singapore, $36(1), 49-57$.

Liu, K., Lu, Y., Lee, J. K., Samara, R., Willenberg, R., Sears-Kraxberger, I., . . He, Z. (2010). PTEN deletion enhances the regenerative ability of adult corticospinal neurons. Nat Neurosci, 13(9), 1075-1081. doi: 10.1038/nn.2603

Liu, R.Y. \& Snider, W.D. (2001). Different signaling pathways mediate regenerative versus developmental sensory axon growth. Journal of Neuroscience, 21(17), RC164.

Manczak, M., Mao, P., Nakamura, K., Bebbington, C., Park, B., \& Reddy, P. H. (2009). Neutralization of granulocyte macrophage colony-stimulating factor decreases amyloid beta $1-42$ and suppresses microglial activity in a transgenic mouse model of Alzheimer's disease. Hum Mol Genet, 18(20), 3876-3893. doi: $10.1093 / \mathrm{hmg} / \mathrm{ddp} 331$ 
Mangano, E. N., Peters, S., Litteljohn, D., So, R., Bethune, C., Bobyn, J., . . Hayley, S. (2011). Granulocyte macrophage-colony stimulating factor protects against substantia nigra dopaminergic cell loss in an environmental toxin model of Parkinson's disease. Neurobiol Dis, 43(1), 99-112. doi: 10.1016/j.nbd.2011.02.011

McLay, R.N., Kimura, M., Banks, W.A. \& Kastin, A.J. (1997). Granulocytemacrophage colony-stimulating factor crosses the blood-brain and blood-spinal cord barriers. Brain, 120, 2083-2091.

Muramatsu, R., Ueno, M., \& Yamashita, T. (2009). Intrinsic regenerative mechanisms of central nervous system neurons. Biosci Trends, 3(5), 179-183.

Newbern, J. M., Shoemaker, S. E., \& Snider, W. D. (2009). Taking off the SOCS: cytokine signaling spurs regeneration. Neuron, 64(5), 591-592. doi: 10.1016/j.neuron.2009.11.027

Ousman, S. S., \& David, S. (2001). MIP-1alpha, MCP-1, GM-CSF, and TNF-alpha control the immune cell response that mediates rapid phagocytosis of myelin from the adult mouse spinal cord. $J$ Neurosci, $21(13), 4649-4656$.

Park, H. C., Shim, Y. S., Ha, Y., Yoon, S. H., Park, S. R., Choi, B. H., \& Park, H. S. (2005). Treatment of complete spinal cord injury patients by autologous bone marrow cell transplantation and administration of granulocyte-macrophage colony stimulating factor. Tissue Eng, 11(5-6), 913-922. doi: 10.1089/ten.2005.11.913

Park, K. K., Liu, K., Hu, Y., Smith, P. D., Wang, C., Cai, B., . . He, Z. (2008). Promoting axon regeneration in the adult CNS by modulation of the 
PTEN/mTOR pathway. Science, 322(5903), 963-966. doi: $10.1126 /$ science. 1161566

Peterson, W. M., Wang, Q., Tzekova, R., \& Wiegand, S. J. (2000). Ciliary neurotrophic factor and stress stimuli activate the Jak-STAT pathway in retinal neurons and glia. JNeurosci, 20(11), 4081-4090.

Qin, H., Holdbrooks, A.T., Liu, Y., Reynolds, S.L., Yanagisawa, L.L. \& Benveniste, E.N. (2012). SOCS3 deficiency promotes M1 macrophage polarization and inflammation. Journal of Immunology.

Reddy, P. H., Manczak, M., Zhao, W., Nakamura, K., Bebbington, C., Yarranton, G., \& Mao, P. (2009). Granulocyte-macrophage colony-stimulating factor antibody suppresses microglial activity: implications for anti-inflammatory effects in Alzheimer's disease and multiple sclerosis. J Neurochem, 111(6), 1514-1528. doi: $10.1111 / \mathrm{j} .1471-4159.2009 .06432 . x$

Richardson, P. M., McGuinness, U. M., \& Aguayo, A. J. (1980). Axons from CNS neurons regenerate into PNS grafts. Nature, 284(5753), 264-265.

Ridwan, S., Bauer, H., Frauenknecht, K., vonPein, H. \& Sommer, C.J. (2012). Distribution of granulocyte macrophage-colony stimulating factor and its receptor $\alpha$-subunit in the adult human brain with specific reference to Alzheimer's disease. Journal of Neural Transmission.

Rotshenker, S. (2011). Wallerian degeneration: the innate-immune response to traumatic nerve injury. J Neuroinflammation, 8,109 . doi: 10.1186/1742-2094-8-109 
Schallenberg, M., Charalambous, P. \& Thanos, S. (2009). GM-CSF regulates the ERK1/2 pathways and protects injured retinal ganglion cells from induced death. Experimental Eye Research, 89(5), 665-677.

Shibuya, S., Yamamoto, T., \& Itano, T. (2009). Glial and axonal regeneration following spinal cord injury. Cell Adh Migr, 3(1), 99-106.

Silver, J., \& Miller, J. H. (2004). Regeneration beyond the glial scar. Nat Rev Neurosci, $5(2), 146-156$. doi: $10.1038 / \mathrm{nrn} 1326$

Smith, P. D., Sun, F., Park, K. K., Cai, B., Wang, C., Kuwako, K., . . He, Z. (2009). SOCS3 deletion promotes optic nerve regeneration in vivo. Neuron, 64(5), 617623. doi: 10.1016/j.neuron.2009.11.021

Sofroniew, M. V. (2009). Molecular dissection of reactive astrogliosis and glial scar formation. Trends Neurosci, 32(12), 638-647. doi: 10.1016/j.tins.2009.08.002

Stosser, S., Schweizerhof, M., \& Kuner, R. (2011). Hematopoietic colony-stimulating factors: new players in tumor-nerve interactions. $J$ Mol Med (Berl), 89(4), 321329. doi: $10.1007 / \mathrm{s} 00109-010-0697-\mathrm{z}$

Sun, F., \& He, Z. (2010). Neuronal intrinsic barriers for axon regeneration in the adult CNS. Curr Opin Neurobiol, 20(4), 510-518. doi: 10.1016/j.conb.2010.03.013

Sun, F., Park, K. K., Belin, S., Wang, D., Lu, T., Chen, G., . . He, Z. (2011). Sustained axon regeneration induced by co-deletion of PTEN and SOCS3. Nature. doi: $10.1038 /$ nature 10594

Tanaka, E. M., \& Ferretti, P. (2009). Considering the evolution of regeneration in the central nervous system. Nat Rev Neurosci, 10(10), 713-723. doi: 10.1038/nrn2707 
Tavazoie, S.F., Alvarez, V.A., Ridenour, D.A., Kwiatkowski, D.J. \& Sabatini, B.L. (2005). Regulation of neuronal morphology and function by the tumor suppressors Tsc1 and Tsc2. Nature Neuroscience, 8(12), 1727-1734.

Thuret, S., Moon, L. D., \& Gage, F. H. (2006). Therapeutic interventions after spinal cord injury. Nat Rev Neurosci, 7(8), 628-643. doi: 10.1038/nrn1955

Vassiliou, I., Lolis, E., Nastos, C., Tympa, A., Theodosopoulos, T., Dafnios, N., . . . Smyrniotis, V. (2010). The combined effect of erythropoietin and granulocyte macrophage colony stimulating factor on liver regeneration after major hepatectomy in rats. World J Surg Oncol, 8, 57. doi: 10.1186/1477-7819-8-57

Yiu, G., \& He, Z. (2006). Glial inhibition of CNS axon regeneration. Nat Rev Neurosci, 7(8), 617-627. doi: $10.1038 / \mathrm{nrn} 1956$

Yoon, S. H., Shim, Y. S., Park, Y. H., Chung, J. K., Nam, J. H., Kim, M. O., . . Ha, Y. (2007). Complete spinal cord injury treatment using autologous bone marrow cell transplantation and bone marrow stimulation with granulocyte macrophagecolony stimulating factor: Phase I/II clinical trial. Stem Cells, 25(8), 2066-2073. doi: 10.1634/stemcells.2006-0807

Zhan, Y., Xu, Y. \& Lew, A.M. (2012). The regulation of the development and function of dendritic cell subsets by GM-CSF: More than a hematologic growth factor. Molecular Immunology, 52(1), 30-37. 